\title{
Capacity of Quantum Private Information Retrieval with Colluding Servers
}

\author{
Seunghoan Song, Member, IEEE, and Masahito Hayashi, Fellow, IEEE
}

\begin{abstract}
Quantum private information retrieval (QPIR) is a protocol in which a user retrieves one of multiple files from n non-communicating servers by downloading quantum systems without revealing which file is retrieved. As variants of QPIR with stronger security requirements, symmetric QPIR is a protocol in which no other files than the target file are leaked to the user, and t-private QPIR is a protocol in which the identity of the target file is kept secret even if at most $t$ servers may collude to reveal the identity. The QPIR capacity is the maximum ratio of the file size to the size of downloaded quantum systems, and we prove that the symmetric t-private QPIR capacity is $\min \{1,2(\mathrm{n}-\mathrm{t}) / \mathrm{n}\}$ for any $1 \leq \mathrm{t}<\mathrm{n}$. We construct a capacity-achieving QPIR protocol by the stabilizer formalism and prove the optimality of our protocol. The proposed capacity is greater than the classical counterpart.
\end{abstract}

\section{INTRODUCTION}

\section{A. Classical and quantum private information retrieval}

When a user retrieves information from databases, it is often required to protect the privacy of the user. Private information retrieval (PIR) is the task in which a user retrieves one of $f$ files from $n$ non-communicating servers without revealing to each individual server which file is downloaded. A PIR protocol is constructed by sending queries from the user to the servers and downloading answers in the opposite direction. Chor, Goldreich, Kushilevitz, and Sudan [1] originally considered this problem for one server and proved that the optimal communication complexity is linear with respect to the combined total size of all files, i.e., downloading all files in the server is optimal. They also considered a PIR protocol with multiple servers, and along with the following works [2]-[4], the communication complexity has been significantly improved. Furthermore, when the protocol has multiple servers and the file size is allowed to be arbitrarily large, Sun and Jafar [5] defined the PIR capacity as the maximum rate of the

This article was presented in part at Proceedings of 2020 IEEE International Symposium on Information Theory [47.

S. Song is with Graduate school of Mathematics, Nagoya University, Nagoya, 464-8602, Japan (e-mail: m17021a@math.nagoya-u.ac.jp).

M. Hayashi is with Shenzhen Institute for Quantum Science and Engineering, Southern University of Science and Technology, Shenzhen, 518055 , China, Guangdong Provincial Key Laboratory of Quantum Science and Engineering, Southern University of Science and Technology, Shenzhen 518055, China, Shenzhen Key Laboratory of Quantum Science and Engineering, Southern University of Science and Technology, Shenzhen 518055, China, and Graduate School of Mathematics, Nagoya University, Nagoya, 464-8602, Japan (e-mail:hayashi@sustech.edu.cn).

SS is supported by JSPS Grant-in-Aid for JSPS Fellows No. JP20J11484 and Lotte Foundation Scholarship. $\mathrm{MH}$ is supported in part by Guangdong Provincial Key Laboratory (Grant No. 2019B121203002), a JSPS Grant-inAids for Scientific Research (A) No.17H01280 and for Scientific Research (B) No.16KT0017, and Kayamori Foundation of Information Science Advancement. file size over the download size. Although the communication complexity counts the cost of two-way communication between the user and the servers, the PIR capacity counts only the download size since the upload (query) size can be neglected by reusing the same query many times for retrieving a large file. The method of downloading all files asserts that the PIR capacity is greater than $1 / \mathrm{f}$, and the paper [5] proved that the PIR capacity is $(1-1 / \mathrm{n}) /\left(1-(1 / \mathrm{n})^{\mathrm{f}}\right)$, which approaches 1 as the number of servers $n$ increases. PIR capacities have also been derived in many other settings [6]-[18].

Quantum PIR (QPIR) has also been studied [20]-[29] as a method to overcome the limitations of the classical PIRs, even when the file is composed of classical information. When two-way quantum communication is allowed, Le Gall [23] proposed a one-server QPIR protocol whose communication complexity is the square root of the size of all files. Song and Hayashi [27], [28] discussed the QPIR capacity, which is the maximum ratio of the file size to the size of the downloaded quantum systems when the upload from the user to the servers is classical, the download is quantum, and the servers share entanglement before the protocol starts. They showed that the QPIR capacity is 1 and proposed a capacity-achieving QPIR protocol with only two servers. Their approach clearly differs from the other papers [22]-[26], which considered the one-server QPIR, and the papers [20]-[26], which considered the communication complexity when twoway quantum communication is allowed.

In PIR, the user may obtain some information on the $\mathrm{n}-1$ non-targeted files. Therefore, it is desirable to consider PIR with server secrecy in which the user also obtains no information other than the target file. PIR with the server secrecy is called symmetric PIR, which is also called oblivious transfer [30] in the one-server case. In the classical case, the paper [31] proved that shared randomness among servers is necessary to construct symmetric PIR protocols even with multiple servers. Assuming shared randomness among servers, the paper [7] derived that symmetric PIR capacity is $1-\mathrm{n}^{-1}$. In the quantum case, the paper [21] proved that symmetric QPIR can be implemented if two-way quantum communication is available. The paper [27] showed that symmetric QPIR is also possible with the classical upload, quantum download, and shared entanglement, and derived that the symmetric QPIR capacity is 1 .

Furthermore, one critical weakness of multi-server PIR is the assumption of no communication among servers. To relieve this assumption, PIR has also been studied in the case where some of the servers may communicate and collude to reveal the user's request. t-Private PIR is PIR with stronger user secrecy, 
TABLE I: Capacities of classical and quantum PIRs

\begin{tabular}{|c|c|c|}
\hline & Classical PIR Capacity & Quantum PIR Capacity \\
\hline PIR & $\frac{1-\mathrm{n}^{-1}}{1-\mathrm{n}^{-\mathrm{f}}}[5]$ & $1 \mp[27]$ \\
\hline Symmetric PIR & $1-\frac{1}{\mathrm{n}}[7]^{\dagger}$ & $1 \ddagger[27]$ \\
\hline t-Private PIR & $\frac{1-\mathrm{t} / \mathrm{n}}{1-(\mathrm{t} / \mathrm{n})^{\mathrm{f}}}[8]$ & $\min \left\{1, \frac{2(\mathrm{n}-\mathrm{t})}{\mathrm{n}}\right\} \ddagger$ \\
\hline $\begin{array}{c}\text { t-Private } \\
\text { symmetric PIR }\end{array}$ & $\frac{\mathrm{n}-\mathrm{t}}{\mathrm{n}}[16] \dagger$ & [This paper] \\
\hline
\end{tabular}

$* \mathrm{n}, \mathrm{f}$ : the numbers of servers and files, respectively.

$\dagger$ Shared randomness among servers is necessary.

$\ddagger$ Capacities are derived with the strong converse bounds.

called user t-secrecy or t-privacy, in which the identity of the retrieved file is unknown to any collection of $t$ servers. In the classical case, the paper [8] proved that the $t$-private PIR capacity is $(1-(\mathrm{t} / \mathrm{n})) /\left(1-(\mathrm{t} / \mathrm{n})^{\mathrm{f}}\right)$ for any $1 \leq \mathrm{t}<\mathrm{n}$. Assuming shared randomness among servers, the paper [16] derived that the symmetric t-private PIR capacity is $(n-t) / n$. In the quantum case, the paper [28] proved that the symmetric $(n-1)$-private QPIR capacity is $2 / n$ for an even number of servers $n$, but it has not been proved for $1 \leq \mathrm{t}<\mathrm{n}-1$.

\section{B. Contribution}

As a generalization of the QPIR capacities in [27], [28], we derive the symmetric t-private QPIR capacity for any $t$ less than the number of servers $n$. Similar to the papers [27] and [28], we define the QPIR model as follows: a user retrieves one of $f$ files from $n$ non-communicating servers, each of which contains the whole classical file set; the servers share an entangled state before the protocol starts; the user uploads classical queries and downloads quantum systems; and the user decodes the target file by quantum measurement. The capacity is defined with four security parameters: error probability, server secrecy, user t-secrecy, and upload cost. As a main result, we prove that the symmetric t-private QPIR capacity is $\min \{1,2(n-t) / n\}$ for $1 \leq t<n$. Especially, for $1 \leq \mathrm{t} \leq \mathrm{n} / 2$, the capacity is 1 even if we require the strongest security condition in which the protocol has zeroerror, perfect user t-secrecy, and perfect server secrecy. For the proof, we construct the capacity-achieving protocol by stabilizer formalism and present the tight upper bounds of the QPIR capacity for $1 \leq \mathrm{t} \leq \mathrm{n} / 2$ and $\mathrm{n} / 2<\mathrm{t}<\mathrm{n}$, respectively.

The derived quantum capacity is strictly greater than the classical symmetric t-private PIR capacity $(n-t) / n$ in [16] (see Table @), and when more than half of the servers collude (i.e., $n / 2 \leq t$ ), the derived quantum capacity is exactly twice the classical capacity. In addition, compared to the classical tprivate PIR capacity $(1-t / n) /\left(1-(t / n)^{f}\right)$ [8], our quantum capacity is greater when $t<n / 2$ or $(n / t)^{f}>2$, where the latter inequality is satisfied when the number of files $f$ is large enough.

Our result implies that symmetric $\lfloor n / 2\rfloor$-private QPIR can be constructed without sacrificing any communication efficiency since the capacity is 1 for $1 \leq t \leq n / 2$. Moreover,
QPIR with more servers may obtain stronger secrecy against collusion. This result contrasts the result [27] that symmetric (1-private) QPIR has no benefit of increasing the number of servers because a two-server protocol achieves the capacity for 1-private QPIR.

\section{Outline of protocol construction}

Our protocol is constructed by the stabilizer formalism. Given a subspace $\mathrm{V}$ of an even-dimensional vector space over a finite field $\mathbb{F}_{q}$, let $\mathrm{V}^{\perp_{J}}$ be its orthogonal space with respect to the symplectic bilinear form. In the stabilizer formalism, the stabilizer is described by a subspace $\mathrm{V}$ such that $\mathrm{V} \subset \mathrm{V}^{\perp_{J}}$, and the state is prepared on the stabilized subspace. When the Weyl operator $\tilde{\mathbf{W}}(\mathbf{s}, \mathbf{t}):=\mathbf{X}(\mathbf{s}) \mathbf{Z}(\mathbf{t})$ with vectors $\mathbf{s}, \mathbf{t}$ is applied, an appropriate quantum measurement in the decoding process outputs the outcome $(\mathbf{s}, \mathbf{t})+\mathrm{V}^{\perp_{J}}$, which is partial information of the Weyl operator. With this fact, we design our QPIR protocol so that the servers share an entangled state on the stabilized subspace, the servers apply Weyl operators depending on the queries and files, and the user performs the measurement to obtain the target file. Here, for guaranteeing security, we choose the subspace $\mathrm{V}$ and the queries to satisfy the following three conditions.

1) The queries to any t servers are independent of the user's request (for user secrecy).

2) When the Weyl operator applied by the servers is $\tilde{\mathbf{W}}(\mathbf{s}, \mathbf{t})$ on the whole composite system, the target file information has a one-to-one correspondence with the value $(\mathbf{s}, \mathbf{t})+$ $\mathrm{V}^{\perp_{J}}$ (for correctness).

3) The information of other files is in $\mathrm{V}^{\perp_{J}}$ (for server secrecy).

The main difficulty of the protocol construction is to find an appropriate vector space $\mathrm{V}$ which satisfies the properties 1), 2 ), and 3). The problem reduces to the search of a symplectic matrix with a linear independence condition in row vectors and a symplectic orthogonality condition in column vectors. We concretely construct a symplectic matrix satisfying those conditions.

Our QPIR protocol utilizes a multipartite entangled state whereas the QPIR protocols in [27], [28] are constructed from two well-known bipartite protocols, dense coding [32] and quantum teleportation [33]. The paper [27] constructed a simple QPIR protocol by modifying the dense coding protocol, and the paper [28] constructed an $(n-1)$-private QPIR protocol by combining quantum teleportation and dense coding. In contrast, our QPIR protocol utilizes a multipartite version of dense coding under the stabilizer formalism. The proposed protocol includes the protocol in [27] as an example of symmetric 1-private QPIR protocols.

\section{Organization of paper}

The remainder of the paper is organized as follows. Section III reviews the fundamentals of quantum information theory. Section III formally describes the QPIR protocol, defines the QPIR capacity, and presents the main result of the paper. Section IV is the preliminary section for protocol construction. In this section, we define the stabilizer 
formalism, present a communication protocol for classical messages by stabilizer formalism, and give a fundamental lemma for protocol construction. Section $\mathrm{V}$ constructs the capacity-achieving symmetric t-private QPIR protocol. The proposed protocol has no error, perfect user secrecy, and perfect server secrecy. SectionVI presents the converse bounds of the capacity result. We present three upper bounds of the capacity depending on the number of colluding servers and the security parameters. Section VII is the conclusion of the paper.

Terms and notations: The matrix $I_{n}$ denotes the $n \times n$ identity matrix. For a quantum system $\mathcal{H}, I_{\mathcal{H}}$ denotes identity matrix on $\mathcal{H}$. For a random variable $X, \operatorname{Pr}_{X}[f(X)]$ is the probability that $X$ satisfies the condition $f(X)$.

\section{FUNDAMENTALS OF QUANTUM INFORMATION THEORY}

In this section, we briefly introduces the fundamentals of quantum information theory. More detailed introduction can be found at [40], [41].

A quantum system is a Hilbert space $\mathcal{H}$. Throughout this paper, we only consider finite dimensional Hilbert spaces. A quantum state is defined by a density matrix, which is a Hermitian matrix $\rho$ on $\mathcal{H}$ such that

$$
\rho \geq 0, \quad \operatorname{Tr} \rho=1 .
$$

The set of states on $\mathcal{H}$ is written as $\mathcal{S}(\mathcal{H})$. A state $\rho$ is called a pure state if rank $\rho=1$, which can also be described by a unit vector of $\mathcal{H}$. If a state $\rho$ is not a pure state, it is called a mixed state. The state $\rho_{\text {mix }}:=I_{\mathcal{H}} / \operatorname{dim} \mathcal{H}$ is called the completely mixed state. The composite system of two quantum systems $\mathcal{A}$ and $\mathcal{B}$ is given as the tensor product of the systems $\mathcal{A} \otimes \mathcal{B}$. For a state $\rho \in \mathcal{S}(\mathcal{A} \otimes \mathcal{B})$, the reduced state on $\mathcal{A}$ is written as

$$
\rho_{\mathcal{A}}=\operatorname{Tr}_{\mathcal{B}} \rho
$$

where $\operatorname{Tr}_{\mathcal{B}}$ is the partial trace with respect to the system $\mathcal{B}$. A state $\rho \in \mathcal{S}(\mathcal{A} \otimes \mathcal{B})$ is called a separable state if $\rho$ is written as

$$
\rho=\sum_{i} p_{i} \rho_{\mathcal{A}, i} \otimes \rho_{\mathcal{B}, i},
$$

for some distribution $p=\left\{p_{i}\right\}$ and states $\rho_{\mathcal{A}, i} \in \mathcal{S}(\mathcal{A}), \rho_{\mathcal{B}, i} \in$ $\mathcal{S}(\mathcal{B})$. A state $\rho \in \mathcal{S}(\mathcal{A} \otimes \mathcal{B})$ is called an entangled state if it is not separable. A quantum measurement is defined by a positive-operator valued measure (POVM), which is the set of Hermitian matrices $\left\{M_{x}\right\}_{x \in \mathcal{X}}$ on $\mathcal{H}$ such that

$$
M_{x} \geq 0, \quad \sum_{x \in \mathcal{X}} M_{x}=I_{\mathcal{H}}
$$

When the elements of POVM are orthogonal projections, i.e., $M_{x}^{2}=M_{x}$ and $M_{x}^{\dagger}=M_{x}$, we call the POVM a projectionvalued measure $(P V M)$. A quantum operation is described by a trace-preserving completely positive $(T P-C P)$ map $\kappa$, which is a linear map such that

$$
\begin{aligned}
\operatorname{Tr} \kappa(\rho)=1 & \forall \rho \in \mathcal{S}(\mathcal{H}), \\
\kappa \otimes \iota_{\mathbb{C}^{d}}(\rho) \geq 0 & \forall \rho \in \mathcal{S}\left(\mathcal{H} \otimes \mathbb{C}^{d}\right), \quad \forall d \geq 1,
\end{aligned}
$$

where $\iota_{\mathbb{C}^{d}}$ is the identity map on $\mathbb{C}^{d}$. An example of TP-CP maps is the unitary map, which is defined by $\kappa_{U}(\rho)=U \rho U^{*}$ for a unitary matrix $U$.

Next, we define the information measures used in this paper. For a state $\rho$, the von Neumann entropy is defined by $H(\rho):=$ $-\rho \log \rho$. For $\rho \in \mathcal{S}(\mathcal{A} \otimes \mathcal{B})$, we also denote

$$
\begin{aligned}
H(\mathcal{A B})_{\rho} & :=H(\rho), \\
H(\mathcal{A})_{\rho} & :=H\left(\rho_{\mathcal{A}}\right), \\
H(\mathcal{B})_{\rho} & :=H\left(\rho_{\mathcal{B}}\right),
\end{aligned}
$$

where $\rho_{\mathcal{A}}$ and $\rho_{\mathcal{B}}$ are the reduced states on $\mathcal{A}$ and $\mathcal{B}$, respectively. For $\rho \in \mathcal{S}(\mathcal{A} \otimes \mathcal{B} \otimes \mathcal{C})$, the conditional entropy, mutual information, and conditional mutual information are defined by

$$
\begin{aligned}
H(\mathcal{A} \mid \mathcal{B})_{\rho} & :=H(\mathcal{A B})_{\rho}-H(\mathcal{B})_{\rho} \\
I(\mathcal{A} ; \mathcal{B})_{\rho} & :=H(\mathcal{A})_{\rho}-H(\mathcal{A} \mid \mathcal{B})_{\rho} \\
I(\mathcal{A} ; \mathcal{B} \mid \mathcal{C})_{\rho} & :=H(\mathcal{A} \mid \mathcal{C})_{\rho}-H(\mathcal{A} \mid \mathcal{B C})_{\rho}
\end{aligned}
$$

Let $X$ be a random variable with values in $\mathcal{X}$ and a probability distribution $p_{X}=\left\{p_{x} \mid x \in \mathcal{X}\right\}$. When a state $\rho(X)$ on $\mathcal{A}$ depends on the value of $X$, the joint state is written as $\tilde{\rho}(X):=\sum_{x} p_{x}|x\rangle\langle x| \otimes \rho(x)$, which is called a classicalquantum state. For simplicity, we denote

$$
\begin{aligned}
H(\cdot)_{\rho(X)} & :=H(\cdot)_{\tilde{\rho}(X)}, \\
H(\cdot \mid \cdot)_{\rho(X)} & :=H(\cdot \mid \cdot)_{\tilde{\rho}(X)}, \\
I(\cdot ; \cdot)_{\rho(X)} & :=I(\cdot ; \cdot)_{\tilde{\rho}(X),} \\
I(\cdot ; \cdot \mid \cdot)_{\rho(X)} & :=I(\cdot ; \cdot \mid \cdot)_{\tilde{\rho}(X)} \cdot
\end{aligned}
$$

For two distributions $p=\left\{p_{x} \mid x \in \mathcal{X}\right\}$ and $q=\left\{q_{x} \mid x \in\right.$ $\mathcal{X}\}$, the classical relative entropy is defined as

$$
D(p \| q):= \begin{cases}\sum_{x \in \mathcal{X}} p_{x} \log \frac{p_{x}}{q_{x}} & \text { if } \operatorname{supp}(p) \subset \operatorname{supp}(q) \\ \infty & \text { otherwise }\end{cases}
$$

where $\operatorname{supp}(p):=\left\{x \in \mathcal{X} \mid p_{x} \neq 0\right\}$. For two states $\rho$ and $\sigma$ on $\mathcal{H}$, the quantum relative entropy is defined as

$$
D(\rho \| \sigma):=\left\{\begin{array}{ll}
\operatorname{Tr} \rho(\log \rho-\log \sigma) & \text { if } \operatorname{supp}(\rho) \subset \operatorname{supp}(\sigma) \\
\infty & \text { otherwise }
\end{array},\right.
$$

where $\operatorname{supp}(\rho):=\{|x\rangle \in \mathcal{H}|\rho| x\rangle \neq 0\}$.

\section{QPIR MODEL AND MAIN RESULT}

In this section, we formally state the definition of the QPIR protocol, which is illustrated in Fig. 1, and present the main result of the paper. Since the protocol is identically defined as in [27], [28], we review the formal description of the QPIR protocol given in [27], [28]. Then we define the security measures and the capacity of t-private QPIR, and state the main result. 


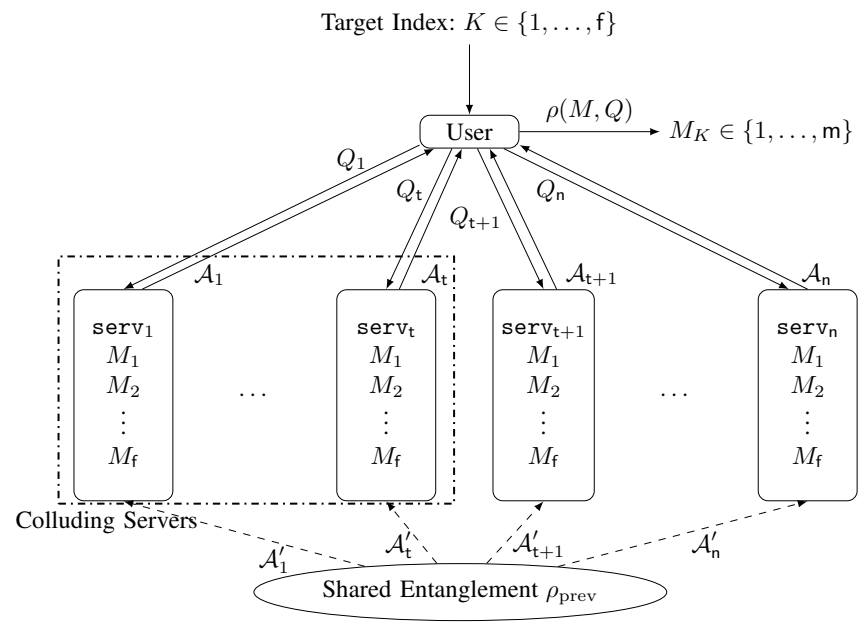

Fig. 1: Quantum private information retrieval protocol, where $\mathrm{t}$ servers collude to know the target index $K$. The user does not know which $t$ servers collude.

\section{A. Formal definition of a QPIR protocol}

The task of t-private QPIR is described as follows. The files $M_{1}, \ldots, M_{\mathrm{f}} \in\{1, \ldots, \mathrm{m}\}$ are uniformly and independently distributed. Each of $\mathrm{n}$ servers $\operatorname{serv}_{1}, \ldots, \operatorname{serv}_{\mathrm{n}}$ contains a copy of all files $M:=\left(M_{1}, \ldots, M_{\mathrm{f}}\right)$. The $\mathrm{n}$ servers are assumed to share an entangled state. A user chooses a file index $K \in\{1, \ldots, \mathrm{f}\}$ uniformly and independently of $M$ in order to retrieve the file $M_{K}$. The task of t-private QPIR is to construct a protocol that allows the user to retrieve $M_{K}$ without revealing $K$ to any collection of $\mathrm{t}$ servers. In the following, we give the formal definition of the QPIR protocol. The concrete construction of our QPIR protocol will be given in Section $\nabla$.

A QPIR protocol is formally defined as follows. Let $\mathcal{A}_{1}^{\prime}, \ldots, \mathcal{A}_{\mathrm{n}}^{\prime}$ be $\mathrm{d}^{\prime}$-dimensional Hilbert spaces. The state of the quantum system $\mathcal{A}_{1}^{\prime} \otimes \cdots \otimes \mathcal{A}_{\mathrm{n}}^{\prime}$ is initialized as $\rho_{\text {prev }}$ and is distributed so that the $s$-th server $\operatorname{serv}_{s}$ contains $\mathcal{A}_{s}^{\prime}$. The user randomly encodes the index $K$ to classical queries $Q_{1}, \ldots, Q_{n}$, i.e.,

$$
\operatorname{Enc}_{\text {user }}(K)=Q=\left(Q_{1}, \ldots, Q_{\mathrm{n}}\right) \in \mathcal{Q}_{1} \times \cdots \times \mathcal{Q}_{\mathrm{n}},
$$

where $\mathcal{Q}_{1}, \ldots, \mathcal{Q}_{\mathrm{n}}$ are finite sets. Then, the user sends $Q_{s}$ to the $s$-th server $\operatorname{serv}_{s}(s=1, \ldots, \mathrm{n})$. Let $\mathcal{A}_{1}, \ldots, \mathcal{A}_{\mathrm{n}}$ be $\mathrm{d}$ dimensional Hilbert spaces and $\mathcal{A}:=\mathcal{A}_{1} \otimes \cdots \otimes \mathcal{A}_{\mathrm{n}}$. After receiving the query $Q_{s}$, the $s$-th server serv $v_{s}$ constructs a TP-CP map $\Lambda_{s}$ from $\mathcal{A}_{s}^{\prime}$ to $\mathcal{A}_{s}$ by the server encoder Enc serv $_{s}$ as

$$
\operatorname{Enc}_{\text {serv }_{s}}\left(Q_{s}, M\right)=\Lambda_{s},
$$

applies $\Lambda_{s}$, and sends $\mathcal{A}_{s}$ to the user. Then, the state on $\mathcal{A}_{1} \otimes \cdots \otimes \mathcal{A}_{\mathrm{n}}$ is written as

$$
\rho(M, Q):=\Lambda_{1} \otimes \cdots \otimes \Lambda_{\mathrm{n}}\left(\rho_{\text {prev }}\right) .
$$

The user decodes the received state by a decoder, which is defined as a $\operatorname{POVM} \operatorname{Dec}(K, Q):=\left\{Y_{K Q}(w) \mid w \in\{1, \ldots, \mathrm{m}\}\right\}$ on $\mathcal{A}_{1} \otimes \cdots \otimes \mathcal{A}_{\mathrm{n}}$ depending on the variables $K$ and $Q$. The user outputs the measurement outcome $W$ as the retrieval result.

Given the numbers of servers $n$ and files $f$, a QPIR protocol of the file size $\mathrm{m}$ is described by

$$
\Psi_{\mathrm{QPIR}}^{(\mathrm{m})}:=\left(\rho_{\text {prev }}, \text { Enc }_{\mathrm{user}}, \text { Enc }_{\mathrm{serv}}, \mathrm{Dec}\right)
$$

of the shared entangled state, user encoder, server encoder, and decoder, where Enc $\mathrm{serv}_{\text {se }}:=\left(\mathrm{Enc}_{\mathrm{serv}_{1}}, \ldots, \mathrm{Enc}_{\mathrm{serv}_{\mathrm{n}}}\right)$. The upload cost, the download cost, and the rate of a QPIR protocol $\Psi_{\mathrm{QPIR}}^{(\mathrm{m})}$ are defined by

$$
\begin{aligned}
& U\left(\Psi_{\mathrm{QPIR}}^{(\mathrm{m})}\right):=\prod_{s=1}^{\mathrm{n}}\left|\mathcal{Q}_{s}\right|, \\
& D\left(\Psi_{\mathrm{QPIR}}^{(\mathrm{m})}\right):=\operatorname{dim} \bigotimes_{s=1}^{\mathrm{n}} \mathcal{A}_{s}=\mathrm{d}^{\mathrm{n}}, \\
& R\left(\Psi_{\mathrm{QPIR}}^{(\mathrm{m})}\right):=\frac{\log \mathrm{m}}{\log D\left(\Psi_{\mathrm{QPIR}}^{(\mathrm{m})}\right)}=\frac{\log \mathrm{m}}{\mathrm{n} \log \mathrm{d}} .
\end{aligned}
$$

\section{B. Security measures of t-private QPIR protocol}

In this paper, we consider the security model that the servers and the user do not deviate from the protocol except for the collusion of servers. In t-private QPIR for $t \in\{1, \ldots, n-1\}$, at most $t$ servers may collect their queries to reveal which file is requested by the user, and the user does not know which servers are colluding. We also consider the server secrecy in which the user only obtains the requested file but no information of the other files.

We evaluate the security of a t-private QPIR protocol $\Phi_{\mathrm{QPIR}}^{(\mathrm{m})}$ by the following security measures. Let $W \in\{1, \ldots, \mathrm{m}\}$ be the protocol output, $M_{k}^{c}$ be the collection of all files except for $M_{k}, \mathrm{~S}_{\mathrm{n}}$ be the symmetric group of $\{1, \ldots, \mathrm{n}\}$, i.e., the set of all permutations on $\{1, \ldots, \mathrm{n}\}$, and $Q_{\pi, \mathrm{t}}:=\left(Q_{\pi(1)}, \ldots, Q_{\pi(\mathrm{t})}\right)$ for $\pi \in \mathrm{S}_{\mathrm{n}}$. The error probability, server secrecy, and user $\mathrm{t}$-secrecy are defined as

$$
\begin{aligned}
P_{\mathrm{err}}\left(\Psi_{\mathrm{QPIR}}^{(\mathrm{m})}\right) & :=\max _{(*)} \operatorname{Pr}\left[W \neq m_{k} \mid M=m, Q=q, K=k\right] \\
S_{\mathrm{serv}}\left(\Psi_{\mathrm{QPIR}}^{(\mathrm{m})}\right) & :=\max _{(*)} I\left(M_{k}^{c} ; \mathcal{A} \mid Q=q, K=k\right)_{\rho(M, q)} \\
S_{\mathrm{user}}^{(\mathrm{t})}\left(\Psi_{\mathrm{QPIR}}^{(\mathrm{m})}\right) & :=\max _{\pi \in \mathrm{S}_{\mathrm{n}}} I\left(K ; Q_{\pi, \mathrm{t}}\right),
\end{aligned}
$$

where the maximum $(*)$ is taken for all $m=$ $\left(m_{1}, \ldots, m_{\mathrm{f}}\right), q \in \mathcal{Q}_{1} \times \cdots \times \mathcal{Q}_{\mathrm{n}}, k \in\{1, \ldots, \mathrm{f}\}$ such that $\operatorname{Pr}[Q=q, K=k] \neq 0$.

The error probability $P_{\mathrm{err}}\left(\Psi_{\mathrm{QPIR}}^{(\mathrm{m})}\right)$ is the worst-case probability that the protocol output $W$ is not the target file of the user. The server secrecy $S_{\mathrm{serv}}\left(\Psi_{\mathrm{QPIR}}^{(\mathrm{m})}\right)$ measures the independence between the non-targeted files and the quantum systems $\mathcal{A}$ that the user obtains. If $S_{\text {serv }}\left(\Psi_{\text {QPIR }}^{(\mathrm{m})}\right)=0$, the user obtains no information of the non-targeted files. The user t-secrecy $S_{\text {user }}^{(\mathrm{t})}\left(\Psi_{\mathrm{QPIR}}^{(\mathrm{m})}\right)$ is defined as the mutual information between the target index $K$ and the queries to any t servers $Q_{\pi, \mathrm{t}}$. If $S_{\text {user }}^{(\mathrm{t})}\left(\Psi_{\mathrm{QPIR}}^{(\mathrm{m})}\right)=0$, any $\mathrm{t}$ servers obtain no information of $K$ even if they collect their queries. These security measures are defined for the worst-case of all files $m$, queries $q$, and the target index $k$. A protocol $\Psi_{\mathrm{QPIR}}^{(\mathrm{m})}$ is a t-private QPIR protocol 
with perfect security if $P_{\mathrm{err}}\left(\Psi_{\mathrm{QPIR}}^{(\mathrm{m})}\right)=S_{\mathrm{user}}^{(\mathrm{t})}\left(\Psi_{\mathrm{QPIR}}^{(\mathrm{m})}\right)=0$ and a symmetric t-private QPIR protocol with perfect security if $P_{\text {err }}\left(\Psi_{\mathrm{QPIR}}^{(\mathrm{m})}\right)=S_{\mathrm{serv}}\left(\Psi_{\mathrm{QPIR}}^{(\mathrm{m})}\right)=S_{\mathrm{user}}^{(\mathrm{t})}\left(\Psi_{\mathrm{QPIR}}^{(\mathrm{m})}\right)=0$.

Remark 1. The server secrecy is also written as

$$
S_{\mathrm{serv}}\left(\Psi_{\mathrm{QPIR}}^{(\mathrm{m})}\right)=\max _{(*)} I\left(M_{k}^{c} ; \mathcal{A} \mid Q=q\right)_{\rho(M, q)} .
$$

This equation follows from $I\left(M_{k}^{c} ; \mathcal{A} \mid Q=q\right)_{\rho(M, q)}=$ $I\left(M_{k}^{c} ; \mathcal{A} \mid Q=q, K=k\right)_{\rho(M, q)}$ which is derived from the independence between $K$ and $\left(M_{k}^{c}, \mathcal{A}\right)$ when $Q=q$ is fixed.

Remark 2. When a QPIR protocol $\Psi_{\mathrm{QPIR}}^{(\mathrm{m})}$ satisfies $P_{\text {err }}\left(\Psi_{\mathrm{QPIR}}^{(\mathrm{m})}\right) \leq \alpha$ and $S_{\text {serv }}\left(\Psi_{\mathrm{QPIR}}^{(\mathrm{m})}\right) \leq \beta$ for sufficiently small $\alpha, \beta \geq 0$, the condition $\operatorname{Pr}[K=k, Q=q] \neq 0$ implies $\operatorname{Pr}[K=i, Q=q]=0$ for any $k \neq i \in\{1, \ldots, \mathrm{f}\}$. Otherwise, we derive a contradiction as follows. If $\operatorname{Pr}[K=$ $k, Q=q] \cdot \operatorname{Pr}[K=i, Q=q] \neq 0$ for some $k \neq i$, the server secrecy $S_{\mathrm{serv}}\left(\Psi_{\mathrm{QPIR}}^{(\mathrm{m})}\right) \leq \beta$ implies $I\left(M_{i}^{c} ; \mathcal{A} \mid Q=q, K=\right.$ $i)_{\rho(M, q)}, I\left(M_{k}^{c} ; \mathcal{A} \mid Q=q, K=k\right)_{\rho(M, q)} \leq \beta$. However, we have the following contradiction

$$
\begin{aligned}
& (1-\alpha) \log \mathrm{m}-h_{2}(\alpha) \stackrel{(a)}{\leq} I\left(M_{k} ; \mathcal{A} \mid Q=q, K=k\right)_{\rho(M, q)} \\
& \stackrel{(b)}{=} I\left(M_{k} ; \mathcal{A} \mid Q=q\right)_{\rho(M, q)} \leq I\left(M_{i}^{c} ; \mathcal{A} \mid Q=q\right)_{\rho(M, q)} \\
& \stackrel{(b)}{=} I\left(M_{i}^{c} ; \mathcal{A} \mid Q=q, K=i\right)_{\rho(M, q)} \leq \beta
\end{aligned}
$$

where $(a)$ is from Fano's inequality and two equalities with $(b)$ are from the independence of $K$ and $\left(M_{1}, \ldots, M_{\mathrm{f}}, \mathcal{A}\right)$ when $Q=q$ is fixed. Since $\beta$ can be chosen to be an arbitrary small number, these inequalities imply that the message size $\mathrm{m}$ is also sufficiently close to zero, which is a contradiction.

\section{C. t-Private QPIR capacity}

When the numbers of servers $n$ and files $f$ are fixed, the t-private QPIR capacity is defined as the optimal rate of the QPIR protocols depending on the security and upload constraints. For any $\alpha \in[0,1)$ and any $\beta, \gamma, \theta \in[0, \infty]$, the asymptotic and exact security-constrained t-private QPIR capacities are defined by

$$
\begin{aligned}
C_{\text {asymp,t }}^{\alpha, \beta, \gamma, \theta} & :=\sup _{\text {20] }} \liminf _{\ell \rightarrow \infty} R\left(\Psi_{\mathrm{QPIR}}^{\left(\mathrm{m}_{\ell}\right)}\right), \\
C_{\text {exact }, \mathrm{t}}^{\alpha, \beta, \gamma, \theta} & :=\sup _{\text {[21] }} \liminf _{\ell \rightarrow \infty} R\left(\Psi_{\mathrm{QPIR}}^{\left(\mathrm{m}_{\ell}\right)}\right),
\end{aligned}
$$

where the supremum is taken for sequences $\left\{m_{\ell}\right\}_{\ell=1}^{\infty}$ such that $\lim _{\ell \rightarrow \infty} \mathrm{m}_{\ell}=\infty$ and sequences $\left\{\Psi_{\mathrm{QPIR}}^{\left(\mathrm{m}_{\ell}\right)}\right\}_{\ell=1}^{\infty}$ of QPIR protocols. and sequences $\left\{\Psi_{\mathrm{QPIR}}^{\left(\mathrm{m}_{\ell}\right)}\right\}_{\ell=1}^{\infty}$ of QPIR protocols to satisfy either (20) or (21) given by

$$
\begin{aligned}
& \limsup _{\ell \rightarrow \infty} P_{\operatorname{err}}\left(\Psi_{\mathrm{QPIR}}^{\left(\mathrm{m}_{\ell}\right)}\right) \leq \alpha, \\
& \limsup _{\ell \rightarrow \infty} S_{\mathrm{serv}}\left(\Psi_{\mathrm{QPIR}}^{\left(\mathrm{m}_{\ell}\right)} \leq \beta,\right. \\
& \limsup _{\ell \rightarrow \infty} S_{\mathrm{user}}^{(\mathrm{t})}\left(\Psi_{\mathrm{QPIR}}^{\left(\mathrm{m}_{\ell}\right)} \leq \gamma,\right. \\
& \limsup _{\ell \rightarrow \infty} \frac{\log U\left(\Psi_{\mathrm{QPIR}}^{\left(\mathrm{m}_{\ell}\right)}\right)}{\log D\left(\Psi_{\mathrm{QPIR}}^{\left(\mathrm{m}_{\ell}\right)}\right)} \leq \theta,
\end{aligned}
$$

and

$$
\begin{aligned}
& P_{\mathrm{err}}\left(\Psi_{\mathrm{QPIR}}^{\left(\mathrm{m}_{\ell}\right)}\right) \leq \alpha, \\
& S_{\mathrm{serv}}\left(\Psi_{\mathrm{QPIR}}^{\left(\mathrm{m}_{\ell}\right)}\right) \leq \beta, \\
& S_{\mathrm{user}}^{(\mathrm{t})}\left(\Psi_{\mathrm{QPIR}}^{\left(\mathrm{m}_{\ell}\right)}\right) \leq \gamma, \\
& \limsup _{\ell \rightarrow \infty} \frac{\log U\left(\Psi_{\mathrm{QPIR}}^{\left(\mathrm{m}_{\ell}\right)}\right)}{\log D\left(\Psi_{\mathrm{QPIR}}^{\left(\mathrm{m}_{\ell}\right)}\right)} \leq \theta .
\end{aligned}
$$

With this definition, we consider symmetric and nonsymmetric capacities at the same time. The capacities $C_{\mathrm{asymp}, \mathrm{t}}^{0, \beta, 0, \theta}$ and $C_{\text {exact,t }}^{0, \beta, 0, \theta}$ are t-private QPIR capacities and $C_{\text {asymp,t }}^{0,0,0, \theta}$ and $C_{\text {exact, }}^{0,0,0, \theta}$ are symmetric t-private QPIR capacities with perfect security. For any $\alpha, \beta, \gamma, \theta$ and $\mathrm{t} \leq \mathrm{t}^{\prime}$, we have the inequalities $C_{\text {exact,t }}^{0,0,0,0} \leq C_{\text {exact,t }}^{\alpha, \beta, \gamma} \leq C_{\text {asymp,t }}^{\alpha, \beta, \gamma, \theta}, C_{\text {asymp,t }}^{\alpha, \beta, \gamma, \theta} \geq C_{\text {asymp,t' }}^{\alpha, \beta, \gamma, \theta}$, and $C_{\text {exact, }}^{\alpha, \beta, \gamma, \theta} \geq C_{\text {exact, } \mathrm{t}^{\prime}}^{\alpha, \beta, \gamma}$ from definition.

\section{Main result}

The following theorem is the main result of the paper.

Theorem 1. The capacity of t-private $Q P I R$ with $\mathrm{n} \geq 2$ servers and $\mathrm{f} \geq 2$ files is derived for any $\alpha \in[0,1)$ and any $\beta, \gamma, \theta \in[0, \infty)$ as follows:

$$
\begin{array}{ll}
C_{\text {asymp,t }}^{\alpha, \beta, \gamma, \theta}=C_{\text {exact,t }}^{\alpha, \beta, \gamma, \theta}=1 & \text { if } 1 \leq \mathrm{t} \leq \frac{\mathrm{n}}{2}, \\
C_{\text {asymp,t }}^{0, \beta, 0, \theta}=C_{\text {exact, } \mathrm{t}}^{\alpha, 0,0, \theta}=\frac{2(\mathrm{n}-\mathrm{t})}{\mathrm{n}} & \text { if } \frac{\mathrm{n}}{2}<\mathrm{t}<\mathrm{n} .
\end{array}
$$

Theorem 1 includes the QPIR capacity without collusion [27] as the case $t=1$. In addition, our result implies that the remarkable result of capacity 1 extends to the case $t \leq n / 2$. Furthermore, since the (1-private) multi-round QPIR capacity is 1 [27], we also obtain the following corollary.

Corollary 1. For $1 \leq \mathrm{t} \leq \mathrm{n} / 2$, the symmetric and nonsymmetric t-private multi-round QPIR capacity is 1 .

When $\mathrm{t} \leq \mathrm{n} / 2$, the capacity decreases but it is twice the symmetric t-private classical PIR capacity $(n-t) / n$ [16] and is still independent of the number of files $f$.

In the remainder of the paper, we prove Theorem 1 by two parts. First, in Section V] we construct a capacity-achieving symmetric t-private QPIR protocol by the stabilizer formalism. To be precise, for $\mathrm{n} / 2 \leq \mathrm{t}<\mathrm{n}$, we construct a protocol with QPIR rate $R\left(\Psi_{\mathrm{QPIR}}^{(\mathrm{m})}\right)=2(\mathrm{n}-\mathrm{t}) / \mathrm{n}$ and perfect security $P_{\text {err }}\left(\Psi_{\text {QPIR }}^{(\mathrm{m})}\right)=S_{\text {serv }}\left(\Psi_{\text {QPIR }}^{(\mathrm{m})}\right)=S_{\text {user }}^{(\mathrm{t})}\left(\Psi_{\text {QPIR }}^{(\mathrm{m})}\right)=0$. For $1 \leq$ $\mathrm{t}<\mathrm{n} / 2$, our $(\mathrm{n} / 2)$-private QPIR protocol also achieves $\mathrm{t}$ private capacity since the protocol achieves rate 1 and the user $(n / 2)$-secrecy guarantees the user t-secrecy. Second, in Section VI we prove the converse bounds. Furthermore, we prove in Appendix A that the capacity result is the same even if we change the definition of the security measures as the average measures for all files $m$, queries $q$, and target indexes $k$.

Remark 3. In the definition of the protocol, we assumed the condition that the target index $K$ and the files $M_{1}, \ldots, M_{\mathrm{f}}$ are chosen uniformly. Indeed, this condition is necessary only for the proof of converse bounds. Even if the distributions 
are arbitrary, the protocol in Section $\nabla$ guarantees that any t-servers obtain no information about $K$ and the user obtains no information of non-targeted files, except for the information obtained from the initial distributions of $K$ and $M_{1}, \ldots, M_{\mathrm{f}}$.

Remark 4. The capacity (23) is derived for the case where any server secrecy $\beta \in[0, \infty)$ is allowed. However, one may notice that for some parameters $(n, t, f)$, the capacity 23 is smaller than the capacity $(1-(\mathrm{t} / \mathrm{n})) /\left(1-(\mathrm{t} / \mathrm{n})^{\mathrm{f}}\right)$ [8] of classical t-private PIR without server secrecy. For instance, when $(\mathrm{n}, \mathrm{t}, \mathrm{f})=(4,3,2)$, the capacity 23) is 0.5 and the capacity in [8] is 0.57 . This follows from the fact that the capacity (23) is derived for finite $\beta$ but the capacity in [8] is derived for the case where $\beta$ is allowed to be infinite.

\section{Preliminaries for Protocol CONSTRuction}

In this section, we give preliminaries for our protocol construction in Section V. Section IV-A introduces the stabilizer formalism and Section IV-B presents a protocol for classical messages constructed defined from the stabilizer formalism. Section IV-C gives a fundamental lemma for the construction of our QPIR protocol.

\section{A. Stabilizer formalism over finite fields}

In this subsection, we introduce the stabilizer formalism for finite fields. Stabilizer formalism gives an algebraic structure for quantum information processing. We use this formalism for the construction of the QPIR protocol. Stabilizer formalism is often used for quantum error-correction. At the end of this subsection (Remark 5), we give a brief review of quantum stabilizer error-correcting codes with the notation introduced in this subsection. More detailed introduction of the stabilizer formalism and stabilizer codes can be found at [34]-[37].

Let $\mathbb{F}_{q}$ be a finite field whose order is a prime power $q=p^{r}$ and $\mathcal{H}$ be a $q$-dimensional Hilbert space with a basis $\{|j\rangle \mid$ $\left.j \in \mathbb{F}_{q}\right\}$. We define $\operatorname{tr} x:=\operatorname{Tr} T_{x} \in \mathbb{F}_{p}$ for $x \in \mathbb{F}_{q}$, where $T_{x} \in \mathbb{F}_{p}^{r \times r}$ denotes the matrix representation of the linear map $y \in \mathbb{F}_{q} \mapsto x y \in \mathbb{F}_{q}$ by identifying the finite field $\mathbb{F}_{q}$ with the vector space $\mathbb{F}_{p}^{r}$. For $a, b \in \mathbb{F}_{q}$, we define two unitary matrices on $\mathcal{H}$

$$
\mathrm{X}(a):=\sum_{j \in \mathbb{F}_{q}}|j+a\rangle\left\langle j\left|, \quad \mathrm{Z}(b):=\sum_{j \in \mathbb{F}_{q}} \omega^{\operatorname{tr} b j}\right| j\right\rangle\langle j|,
$$

where $\omega:=\exp (2 \pi i / p)$. For $\mathbf{a}=\left(a_{1}, \ldots, a_{n}\right), \mathbf{b}=$ $\left(b_{1}, \ldots, b_{n}\right) \in \mathbb{F}_{q}^{n}$, and $\mathbf{w}=(\mathbf{a}, \mathbf{b}) \in \mathbb{F}_{q}^{2 n}$, we define a unitary matrix on $\mathcal{H}^{\otimes n}$

$$
\begin{aligned}
& \tilde{\mathbf{W}}(\mathbf{w})=\tilde{\mathbf{W}}(\mathbf{a}, \mathbf{b}) \\
& :=\mathbf{X}\left(a_{1}\right) \mathbf{Z}\left(b_{1}\right) \otimes \mathbf{X}\left(a_{2}\right) \mathbf{Z}\left(b_{2}\right) \otimes \cdots \otimes \mathbf{X}\left(a_{n}\right) \mathbf{Z}\left(b_{n}\right) .
\end{aligned}
$$

The Heisenberg-Weyl group is defined as

$$
\mathrm{HW}_{q}^{n}:=\left\{c \tilde{\mathbf{W}}(\mathbf{w}) \mid \mathbf{w} \in \mathbb{F}_{q}^{2 n}, c \in \mathbb{C}\right\} .
$$

For $\mathbf{x}, \mathbf{y} \in \mathbb{F}_{q}^{n}$, we denote $\langle\mathbf{x}, \mathbf{y}\rangle:=\operatorname{tr} \sum_{i=1}^{n} x_{i} y_{i} \in \mathbb{F}_{p}$ and define a skew-symmetric matrix $J$ on $\mathbb{F}_{q}^{2 n}$ by

$$
J=\left(\begin{array}{cc}
0 & -I_{n} \\
I_{n} & 0
\end{array}\right)
$$

Since $\mathbf{X}(a) \mathbf{Z}(b)=\omega^{-\operatorname{tr} a b} \mathbf{Z}(b) \mathbf{X}(a)$, for any $(\mathbf{a}, \mathbf{b}),(\mathbf{c}, \mathbf{d}) \in$ $\mathbb{F}_{q}^{2 n}$, we have

$$
\begin{aligned}
\tilde{\mathbf{W}}(\mathbf{a}, \mathbf{b}) \tilde{\mathbf{W}}(\mathbf{c}, \mathbf{d}) & =\omega^{\langle(\mathbf{a}, \mathbf{b}), J(\mathbf{c}, \mathbf{d})\rangle} \tilde{\mathbf{W}}(\mathbf{c}, \mathbf{d}) \tilde{\mathbf{W}}(\mathbf{a}, \mathbf{b}), \\
\tilde{\mathbf{W}}(\mathbf{a}, \mathbf{b}) \tilde{\mathbf{W}}(\mathbf{c}, \mathbf{d}) & =\omega^{\langle\mathbf{b}, \mathbf{c}\rangle} \tilde{\mathbf{W}}(\mathbf{a}+\mathbf{c}, \mathbf{b}+\mathbf{d}) .
\end{aligned}
$$

A commutative subgroup of $\mathrm{HW}_{q}^{n}$ not containing $c I_{\mathcal{H}}^{\otimes n}$ for any $c \neq 0$ is called a stabilizer. A subspace $\mathrm{V}$ of $\mathbb{F}_{q}^{2 n}$ is called self-orthogonal with respect to the bilinear form $\langle\cdot, J \cdot\rangle$ if

$$
\mathrm{V} \subset \mathrm{V}^{\perp_{J}}:=\left\{\mathbf{w} \in \mathbb{F}_{q}^{2 n} \mid\langle\mathbf{v}, J \mathbf{w}\rangle=0 \text { for any } \mathbf{v} \in \mathrm{V}\right\} .
$$

We can define a stabilizer from any self-orthogonal subspace of $\mathbb{F}_{q}^{2 n}$ by the following proposition.

Proposition 1. Let $\mathrm{V}$ be a self-orthogonal subspace of $\mathbb{F}_{q}^{2 n}$. There exists $\left\{c_{\mathbf{v}} \in \mathbb{C} \mid \mathbf{v} \in \mathrm{V}\right\}$ such that

$$
S(\mathrm{~V}):=\left\{\mathbf{W}(\mathbf{v}):=c_{\mathbf{v}} \tilde{\mathbf{W}}(\mathbf{v}) \mid \mathbf{v} \in \mathrm{V}\right\} \subset \mathrm{HW}_{q}^{n}
$$

is a stabilizer.

For the completeness, we give the proof of Proposition 1 in Appendix B.

Proposition 2 ( [37, (8.22), (8.24), Lemma 8.7]). Let $\mathrm{V}$ be a self-orthogonal d-dimensional subspace of $\mathbb{F}_{q}^{2 n}$ and $S(\mathrm{~V})$ be a stabilizer defined from V. For the quotient space $\mathbb{F}_{q}^{2 n} / \mathrm{V}^{\perp_{J}}$, we denote the elements by $[\mathbf{w}]=\mathrm{w}+\mathrm{V}^{\perp_{J}} \in \mathbb{F}_{q}^{2 n} / \mathrm{V}^{\perp_{J}}$. Then, we obtain the following statements.

1) All elements $\mathbf{W}(\mathbf{v}) \in S(\mathrm{~V})$ are simultaneously and uniquely decomposed as

$$
\mathbf{W}(\mathbf{v})=\sum_{[\mathbf{w}] \in \mathbb{F}_{q}^{2 n} / \mathrm{V}^{\perp J}} \omega^{\langle\mathbf{v}, J \mathbf{w}\rangle} P_{[\mathbf{w}]}^{\mathrm{V}} \quad(\forall \mathbf{v} \in \mathrm{V})
$$

with orthogonal projections $\left\{P_{[\mathbf{w}]}^{\mathrm{V}}\right\}$ such that

$$
\begin{aligned}
\sum_{[\mathbf{w}] \in \mathbb{F}_{q}^{2 n} / \mathrm{V}^{\perp J}}^{\mathrm{V}} P_{\left[\mathbf{w}^{\prime}\right]}^{\mathrm{V}} & =0 \text { for any }[\mathbf{w}] \neq\left[\mathbf{w}^{\prime}\right], \\
P_{[\mathbf{w}]}^{\mathrm{V}} & =I_{\mathcal{H} \otimes n} .
\end{aligned}
$$

2) Let $\mathcal{H}_{[\mathbf{w}]}^{\mathrm{V}}:=\operatorname{Im} P_{[\mathbf{w}]}^{\mathrm{V}}$. For any $\mathbf{w}, \mathbf{w}^{\prime} \in \mathbb{F}_{q}^{2 n}$, we have the relation

$$
\mathbf{W}(\mathbf{w}) \mathcal{H}_{\left[\mathbf{w}^{\prime}\right]}^{\mathrm{V}}=\mathcal{H}_{\left[\mathbf{w}+\mathbf{w}^{\prime}\right]}^{\mathrm{V}} .
$$

3) For any $[\mathbf{w}] \in \mathbb{F}_{q}^{2 n} / \mathrm{V}^{\perp_{J}}$,

$$
\operatorname{dim} \mathcal{H}_{[\mathbf{w}]}^{\mathrm{V}}=q^{n-d}
$$

For the completeness, we give the proof of Proposition 2 in Appendix C. As a corollary of Proposition 2, we obtain the following decomposition theorem. We use this decomposition in our protocol construction.

Corollary 2. The quantum system $\mathcal{H}^{\otimes n}$ is decomposed as

$$
\mathcal{H}^{\otimes n}=\bigotimes_{[\mathbf{w}] \in \mathbb{F}_{q}^{2 n} / \mathrm{V}^{\perp J}} \mathcal{H}_{[\mathbf{w}]}^{\mathrm{V}}=\mathcal{W} \otimes \mathbb{C}^{q^{n-d}},
$$

where the system $\mathcal{W}$ is the $q^{d}$-dimensional subspace with the basis $\left\{|[\mathbf{w}]\rangle \mid[\mathbf{w}] \in \mathbb{F}_{q}^{2 n} / \mathrm{V}^{\perp_{J}}\right\}$ such that $\mathcal{H}_{[\mathbf{w}]}^{\mathrm{V}}=$ 
$\left.|[\mathbf{w}]\rangle \otimes \mathbb{C}^{q^{n-d}}:=\{|[\mathbf{w}]\rangle \otimes|v\rangle|| v\rangle \in \mathbb{C}^{q^{n-d}}\right\}$. With this decomposition,

$$
\mathbf{W}(\mathbf{w})\left|\left[\mathbf{w}^{\prime}\right]\right\rangle \otimes \mathbb{C}^{q^{n-d}}=\left|\left[\mathbf{w}+\mathbf{w}^{\prime}\right]\right\rangle \otimes \mathbb{C}^{q^{n-d}} .
$$

Proof: Eq. (33) follows from (30) and (32) and Eq. (34) follows directly from the relation (31).

We also have the following lemma.

Lemma 1. For any $\mathbf{w}, \mathbf{w}^{\prime} \in \mathbb{F}_{q}^{2 n}$, we have

$$
\begin{aligned}
& \mathbf{W}\left(\mathbf{w}^{\prime}\right)\left(|[\mathbf{w}]\rangle\langle[\mathbf{w}]| \otimes I_{q^{n-d}}\right) \mathbf{W}\left(\mathbf{w}^{\prime}\right)^{*} \\
& =\left|\left[\mathbf{w}+\mathbf{w}^{\prime}\right]\right\rangle\left\langle\left[\mathbf{w}+\mathbf{w}^{\prime}\right]\right| \otimes I_{q^{n-d}} .
\end{aligned}
$$

Proof: Let $X:=\mathbf{W}\left(\mathbf{w}^{\prime}\right)\left(|[\mathbf{w}]\rangle\langle[\mathbf{w}]| \otimes I_{q^{n-d}}\right) \mathbf{W}\left(\mathbf{w}^{\prime}\right)^{*}$. Since $X^{2}=X$ and $X^{*}=X$, the matrix $X$ is an orthogonal projection. Since $\left|\left[\mathbf{w}+\mathbf{w}^{\prime}\right]\right\rangle \otimes \mathbb{C}^{q^{n-d}}$ is an invariant subspace of $X$ and $\operatorname{rank} X=\operatorname{dim}\left|\left[\mathbf{w}+\mathbf{w}^{\prime}\right]\right\rangle \otimes \mathbb{C}^{q^{n-d}}=q^{n-d}$, the matrix $X$ is the orthogonal projection onto $\left|\left[\mathbf{w}+\mathbf{w}^{\prime}\right]\right\rangle \otimes \mathbb{C}^{q^{n-d}}$, which implies the lemma.

Remark 5. In terms of quantum stabilizer code, the space $\mathcal{H}_{[\mathbf{0}]}^{\mathrm{V}}=|[\mathbf{0}]\rangle \otimes \mathbb{C}^{q^{n-d}}$ is called the code space, which is the stabilized space by the action of the group $S(\mathrm{~V})$. In other words, from (28), the code space $\mathcal{H}_{[0]}^{\mathrm{V}]}$ is the intersection of eigenspaces of $S(\mathrm{~V})$ with eigenvalue 1. In quantum stabilizer code, a message state is prepared in the code space $\mathcal{H}_{[\mathbf{0}]}^{\mathrm{V}}$. If an error $\mathbf{W}(\mathbf{e})$ is applied, the encoded state on $\mathcal{H}_{[\mathbf{0}]}^{\mathrm{V}}$ is changed to a state on $\mathcal{H}_{[\mathrm{e}]}^{\mathrm{V}}$ by (31). Then, the error correction is preformed by obtaining the identity of the subspace $\mathcal{H}_{[\mathrm{e}]}^{\mathrm{V}}$ by the measurement $\left\{P_{[\mathbf{e}]}^{\mathrm{V}} \mid[\mathbf{e}] \in \mathbb{F}_{q}^{2 n} / \mathrm{V}^{\perp_{J}}\right\}$ on $\mathcal{H}^{\otimes n}$ and performing the recovery operation $\mathbf{W}(-\mathbf{r})$ for some $\mathbf{r} \in[\mathbf{e}]$, which maps the state from $\mathcal{H}_{[\mathbf{e}]}^{\mathrm{V}}$ to $\mathcal{H}_{[\mathbf{0}]}^{\mathrm{V}}$. This error correction is performed correctly if $\mathbf{e}-\mathbf{r} \in \mathrm{V}$ since the combined operation of the error and the correction is $\mathbf{W}(-\mathbf{r}) \mathbf{W}(\mathbf{e})=\mathbf{W}(\mathbf{e}-\mathbf{r})$ and the code space $\mathcal{H}_{[0]}^{\mathrm{V}}$ is invariant with respect to the operation $\mathbf{W}(\mathbf{e}-\mathbf{r})$ if $\mathbf{e}-\mathbf{r} \in \mathrm{V}$. However, if $\mathbf{e}-\mathbf{r} \in \mathrm{V}^{\perp_{J}} \backslash \mathrm{V}$, the error correction may be incorrect. See [34]-[37] for details.

Remark 6. Lemma 1 is equivalent to considering the state on $\mathbb{C}^{q^{n-d}}$ as completely mixed state $\rho_{\text {mix }}=I_{q^{n-d}} / q^{n-d}$. If the state $\rho$ on $\mathbb{C}^{q^{n-d}}$ is not the completely mixed state, there always exists an operation $\mathbf{W}\left(\mathbf{w}^{\prime}\right)$ such that $\rho$ is changed to another state $\rho_{\mathbf{w}^{\prime}}^{\prime}$ as

$\mathbf{W}\left(\mathbf{w}^{\prime}\right)(|[\mathbf{w}]\rangle\langle[\mathbf{w}]| \otimes \rho) \mathbf{W}\left(\mathbf{w}^{\prime}\right)^{*}=\left|\left[\mathbf{w}+\mathbf{w}^{\prime}\right]\right\rangle\left\langle\left[\mathbf{w}+\mathbf{w}^{\prime}\right]\right| \otimes \rho_{\mathbf{w}^{\prime}}^{\prime}$.

For example, we have $\rho \neq \rho_{\mathbf{w}^{\prime}}^{\prime}$ for $[\mathbf{w}]=[\mathbf{0}]$ and some $\mathbf{w}^{\prime} \in$ $\mathrm{V}^{\perp_{J}} \backslash \mathrm{V}$.

\section{B. Communication protocol for classical message by stabilizer formalism}

In this subsection, we propose a communication protocol for classical messages from $n$ players to a receiver. The protocol is constructed by the stabilizer formalism. We will construct our QPIR protocol in Section $\nabla$ by modifying the protocol in this subsection.

In the following protocol, $n$ players encode $\left(a_{1}, b_{1}\right), \ldots$, $\left(a_{n}, b_{n}\right) \in \mathbb{F}_{q}^{2}$ and the receiver decodes

$$
[(\mathbf{a}, \mathbf{b})]=\left[\left(a_{1}, \ldots, a_{n}, b_{1}, \ldots, b_{n}\right)\right] \in \mathbb{F}_{q}^{2 n} / \mathrm{V}^{\perp_{J}},
$$

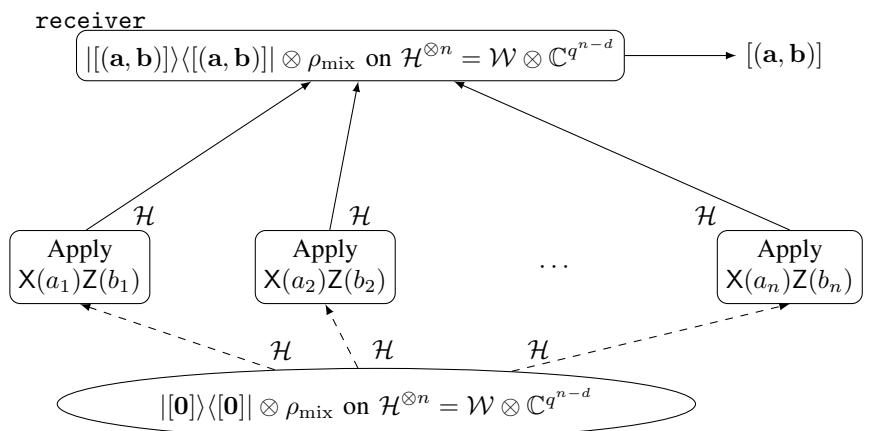

Fig. 2: Protocol 1

where $\mathrm{V}$ is a self-orthogonal subspace of $\mathbb{F}_{q}^{2 n}$. The protocol is depicted in Fig. 2 .

Protocol 1. Let $\mathrm{V}$ be a self-orthogonal d-dimensional subspace of $\mathbb{F}_{q}^{2 n}$ and $S(\mathrm{~V})$ be a stabilizer associated with $\mathrm{V}$. By Corollary 2 we decompose $\mathcal{H}^{\otimes n}$ as

$$
\mathcal{H}^{\otimes n}=\bigotimes_{[\mathbf{w}] \in \mathbb{F}_{q}^{2 n} / \mathrm{V}^{\perp_{J}}} \mathcal{H}_{[\mathbf{w}]}^{\mathrm{V}}=\mathcal{W} \otimes \mathbb{C}^{q^{n-d}} .
$$

1) [Distribution of entangled state] The state of $\mathcal{H}^{\otimes n}=$ $\mathcal{W} \otimes \mathbb{C}^{q^{n-d}}$ is initialized as $|[\mathbf{0}]\rangle\langle[\mathbf{0}]| \otimes \rho_{\text {mix }}$, where $\rho_{\text {mix }}$ is the completely mixed state on $\mathbb{C}^{q^{n-d}}$, i.e., $\rho_{\text {mix }}=$ $I_{q^{n-d}} / q^{n-d}$. The $n$ subsystems of $\mathcal{H}^{\otimes n}$ are distributed to the $n$ players, respectively.

2) [Message encoding] For each $s \in\{1, \ldots, n\}$, the player $s$ applies $\mathrm{X}\left(a_{s}\right) \mathrm{Z}\left(b_{s}\right)$ to the distributed system $\mathcal{H}$ and sends the system $\mathcal{H}$ to the receiver.

3) [Message decoding] The receiver applies the PVM $\mathbf{M}^{\mathrm{V}}=\left\{P_{[\mathbf{w}]}^{\mathrm{V}} \mid[\mathbf{w}] \in \mathbb{F}_{q}^{2 n} / \mathrm{V}^{\perp_{J}}\right\}$ on $\mathcal{H}^{\otimes n}$, where $[\mathbf{w}]$ is the measurement outcome associated with $P_{[\mathbf{w}]}^{\mathrm{V}}$.

In the above protocol, the receiver receives the state $|[(\mathbf{a}, \mathbf{b})]\rangle\langle[(\mathbf{a}, \mathbf{b})]| \otimes \rho_{\text {mix }}$ by Lemma 1 Thus, the receiver obtains

$$
[(\mathbf{a}, \mathbf{b})]=\left[\left(a_{1}, \ldots, a_{n}, b_{1}, \ldots, b_{n}\right)\right] \in \mathbb{F}_{q}^{2 n} / \mathrm{V}^{\perp_{J}}
$$

as the measurement outcome. Note that the receiver can retrieve no more information than $[(\mathbf{a}, \mathbf{b})]$ from the state $|[(\mathbf{a}, \mathbf{b})]\rangle\langle[(\mathbf{a}, \mathbf{b})]| \otimes \rho_{\text {mix }}$. However, if the initial state of $\mathbb{C}^{q^{n-d}}$ is not $\rho_{\text {mix }}$ in Step 1, the receiver may obtain more information about $(\mathbf{a}, \mathbf{b})$ than $[(\mathbf{a}, \mathbf{b})]$ since the final state on $\mathbb{C}^{q^{n-d}}$ may depend on $(\mathbf{a}, \mathbf{b})$. See Remark 6 for more detail.

Protocol 11 is a generalization of the two-sum communication protocol [28, Protocol III.1]. In the two-sum communication protocol, two players share a maximally entangled state and have two-bit classical messages $(a, b),(c, d) \in \mathbb{F}_{2}^{2}$, respectively. Each player applies $\mathbf{X}(a) \mathbf{Z}(b)$ and $\mathbf{X}(c) \mathbf{Z}(d)$ on each entangled system and sends the system to the receiver. Finally, the receiver obtains the sum $(a+c, b+d)$. This protocol is a special case of Protocol 1 since the maximally entangled state is the state $|[\mathbf{0}]\rangle\langle[\mathbf{0}]| \otimes \rho_{\text {mix }}$ by choosing $\mathrm{V}=\operatorname{span}\left\{(1,1,0,0)^{\top},(0,0,1,1)^{\top}\right\}$ with $(n, d)=(2,2)$.

In Section $\mathrm{V}$, we will construct our QPIR protocol by modifying Protocol 11. For fulfilling the QPIR task, we will 
choose a suitable self-orthogonal subspace $\mathrm{V}$ and design the query structure in Section $\mathrm{V}$

\section{Fundamental lemma for protocol construction}

In this subsection, we prepare a fundamental lemma for the QPIR protocol construction. Our QPIR protocol will use the self-orthogonal subspace $\mathrm{V}$ defined from the lemma in this subsection. In the statement of the lemma, we use an extension of a finite field [38], [39]. Let $\mathbb{F}$ be a subfield of $\mathbb{G}$, i.e., $\mathbb{G}$ is an extension field of $\mathbb{F}$. For $\alpha \in \mathbb{G}$, the smallest field containing $\mathbb{F}$ and $\alpha$ is denoted by $\mathbb{F}(\alpha)$. We denote by $\mathbb{F}\left(\alpha_{1}, \ldots, \alpha_{n}\right)$ the field defined recursively by the relation $\mathbb{F}\left(\alpha_{1}, \ldots, \alpha_{k}\right)=$ $\left[\mathbb{F}\left(\alpha_{1}, \ldots, \alpha_{k-1}\right)\right]\left(\alpha_{k}\right)$.

The lemma is given as follows.

Lemma 2. Let $n$, $t$ be positive integers such that $n / 2 \leq t<n$. Let $q^{\prime}$ be an arbitrary prime power, $\mathbb{F}_{q^{\prime}}$ be the finite field of order $q^{\prime}$, and $\mathbb{F}_{q}$ be an extension field of $\mathbb{F}_{q^{\prime}}$ such that $\mathbb{F}_{q}=$ $\mathbb{F}_{q^{\prime}}\left(\alpha_{1}, \ldots, \alpha_{n+2 t-2}\right)$, where $\alpha_{i} \notin \mathbb{F}_{q^{\prime}}\left(\alpha_{1}, \ldots, \alpha_{i-1}\right)$ for any $i$. There exist $2 t$ linearly independent vectors $\mathbf{v}_{1}, \ldots, \mathbf{v}_{2 t} \in$ $\mathbb{F}_{q}^{2 n}$ satisfying the following conditions.

(a) Let $\mathbf{w}_{1}, \ldots, \mathbf{w}_{2 n}$ be the row vectors of the matrix $D=\left(\mathbf{v}_{1}, \ldots, \mathbf{v}_{2 t}\right) \in \mathbb{F}_{q}^{2 n \times 2 t}$. Then, $\mathbf{w}_{\pi(1)}, \ldots, \mathbf{w}_{\pi(t)}, \mathbf{w}_{\pi(1)+n}, \ldots, \mathbf{w}_{\pi(t)+n}$ are linearly independent for any permutation $\pi$ in $\mathrm{S}_{n}$.

(b) $\left\langle\mathbf{v}_{i}, J \mathbf{v}_{j}\right\rangle=0$ for any $i \in\{1, \ldots, 2 n-2 t\}$ and any $j \in\{1, \ldots, 2 t\}$.

The proof of Proposition 2 is given in Appendix D.

Many studies in classical information theory have already studied the matrices $D \in \mathbb{F}_{q}^{n \times t}$ whose arbitrary $t(\leq n)$ row vectors are linearly independent, which is similar to condition (a) of Lemma 2 . For instance, matrices of this kind have been studied as a generator matrix of the maximum distance separable (MDS) codes [45] and have been widely used in the construction of secure communication protocols, e.g., classical private information retrievals [8], [9], [15], wiretap channel II [42], and secure network coding [43], [44].

\section{CONSTRUCTION OF QPIR PROTOCOL WITH COLLUDING SERVERS}

In this section, we construct the capacity-achieving QPIR protocol for $n \geq 2$ servers, $f \geq 2$ files, and $n / 2 \leq t<n$ colluding servers. For collusion of $1 \leq t<n / 2$ servers, our protocol for $\mathrm{t}=\mathrm{n} / 2$ is the capacity-achieving protocol.

\section{A. Construction of protocol}

We construct our QPIR protocol by modifying Protocol 1 . which is defined with a self-orthogonal subspace V. Similar to Protocol 1, in our QPIR protocol, $\mathrm{n}$ servers encode $\left(a_{1}, b_{1}\right), \ldots,\left(a_{\mathrm{n}}, b_{\mathrm{n}}\right) \in \mathbb{F}_{q}^{2}$ and a user receives $[(\mathbf{a}, \mathbf{b})]=$ $\left[\left(a_{1}, \ldots, a_{\mathrm{n}}, b_{1}, \ldots, b_{\mathrm{n}}\right)\right] \in \mathbb{F}_{q}^{2 \mathrm{n}} / \mathrm{V}^{\perp_{J}}$ but no more information of $(\mathbf{a}, \mathbf{b})$. For guaranteeing the correctness and the secrecies of our protocol, we will choose a self-orthogonal subspace $\mathrm{V}$ by Lemma 2 and design the query structure so that

1) the user generates queries depending on the file index $k$, while any $\mathrm{t}$ queries are independent of $k$,

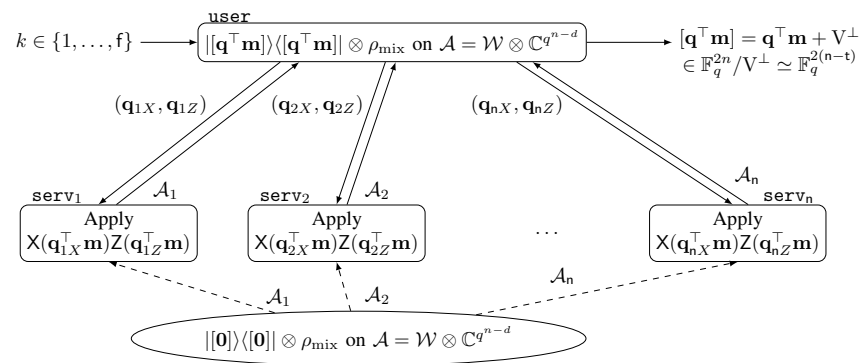

Fig. 3: t-Private QPIR protocol. $\mathbf{m}=\left(\mathbf{m}_{1}^{\top}, \ldots, \mathbf{m}_{k}^{\top}\right)^{\top}$ and $\mathbf{q}=\left(\mathbf{q}_{1 X}^{\top}, \ldots, \mathbf{q}_{\mathrm{n} X}^{\top}, \mathbf{q}_{1 Z}^{\top}, \ldots, \mathbf{q}_{\mathrm{n} Z}^{\top}\right)^{\top}$ are the collections of files and queries, respectively.

2 ) the $s$-th server encodes $\left(a_{i}, b_{i}\right)$ depending on the query and the files, and

3) the user's received message $[(\mathbf{a}, \mathbf{b})]=(\mathbf{a}, \mathbf{b})+\mathrm{V}^{\perp}$ is identical to the $k$-th file.

Our QPIR protocol is described as follows.

Protocol 2. Let $\mathrm{n} \geq 2, \mathrm{f} \geq 2$, and $\mathrm{n} / 2 \leq \mathrm{t}<\mathrm{n}$. The files are $2(\mathrm{n}-\mathrm{t})$-dimensional vectors $\mathbf{m}_{1}, \ldots, \mathbf{m}_{\mathrm{f}} \in \mathbb{F}_{q}^{2(\mathrm{n}-\mathrm{t})}$. Each of $\mathrm{n}$ servers contains a copy of all files $\mathbf{m}:=\left(\mathbf{m}_{1}^{\top}, \ldots, \mathbf{m}_{k}^{\top}\right)^{\top} \in$ $\mathbb{F}_{q}^{2(\mathrm{n}-\mathrm{t}) \mathrm{f}}$. The index of the user's target file is $k$, i.e., the user retrieves $\mathbf{m}_{k}$.

We choose a self-orthogonal vector space $\mathrm{V}$ and introduce some notations as follows. Let $q^{\prime}$ be an arbitrary prime power, $\mathbb{F}_{q^{\prime}}$ be the finite field of order $q^{\prime}$, and $\mathbb{F}_{q}$ be an extension field of $\mathbb{F}_{q^{\prime}}$ such that $\mathbb{F}_{q}=\mathbb{F}_{q^{\prime}}\left(\alpha_{1}, \ldots, \alpha_{n+2 t-2}\right)$, where $\alpha_{i} \notin$ $\mathbb{F}_{q^{\prime}}\left(\alpha_{1}, \ldots, \alpha_{i-1}\right)$ for any $i$. We choose a basis $\mathbf{v}_{1}, \ldots, \mathbf{v}_{2 n}$ of $\mathbb{F}_{q}^{2 n}$ such that the first $2 \mathrm{t}$ vectors $\mathbf{v}_{1}, \ldots, \mathbf{v}_{2 \mathrm{t}}$ satisfy the conditions of Lemma 2. Let $\mathrm{V}:=\operatorname{span}\left\{\mathbf{v}_{1}, \mathbf{v}_{2}, \ldots, \mathbf{v}_{2 \mathrm{n}-2 \mathrm{t}}\right\} \subset \mathbb{F}_{q}^{2 \mathrm{n}}$. Then, from condition (b) of Lemma 2 the subspace $\mathrm{V}$ is selforthogonal with respect to $\langle\cdot, J \cdot\rangle, \mathrm{V}^{\perp J}=\operatorname{span}\left\{\mathbf{v}_{1}, \ldots, \mathbf{v}_{2 \mathrm{t}}\right\}$ and the quotient space $\mathbb{F}_{q}^{2 n} / \mathrm{V}^{\perp_{J}}$ is written as

$\mathbb{F}_{q}^{2 n} / \mathrm{V}^{\perp_{J}}=\left\{[\mathbf{w}]:=\mathbf{w}+\mathrm{V}^{\perp_{J}} \mid \mathbf{w} \in \operatorname{span}\left\{\mathbf{v}_{2 \mathrm{t}+1}, \ldots, \mathbf{v}_{2 \mathbf{n}}\right\}\right\}$.

Let

$$
\begin{aligned}
& D_{1}:=\left(\begin{array}{llll}
\mathbf{v}_{1} & \mathbf{v}_{2} & \cdots & \mathbf{v}_{2 \mathrm{t}}
\end{array}\right) \in \mathbb{F}_{q}^{2 \mathrm{n} \times 2 \mathrm{t}} \\
& D_{2}:=\left(\begin{array}{llll}
\mathbf{v}_{2 \mathrm{t}+1} & \mathbf{v}_{2 \mathrm{t}+2} & \cdots & \mathbf{v}_{2 \mathrm{n}}
\end{array}\right) \in \mathbb{F}_{q}^{2 \mathrm{n} \times 2(\mathrm{n}-\mathrm{t})} .
\end{aligned}
$$

We assume that the vectors $\mathbf{v}_{1}, \ldots, \mathbf{v}_{2 n}$ are publicly known to the user and all servers. With this choice of the selforthogonal space $\mathrm{V}$ and vectors $\mathbf{v}_{1}, \ldots, \mathbf{v}_{2 n}$, the protocol works as follows.

1) [Distribution of Entangled State] Let $\mathcal{A}_{1}, \ldots, \mathcal{A}_{\mathrm{n}}$ be qdimensional Hilbert spaces. From Corollary 2 the quantum system $\mathcal{A}:=\mathcal{A}_{1} \otimes \cdots \otimes \mathcal{A}_{\mathrm{n}}$ is decomposed as $\mathcal{A}=$ $\mathcal{W} \otimes \mathbb{C}^{q^{2 \mathrm{t}-\mathrm{n}}}$, where $\mathcal{W}=\operatorname{span}\left\{|[\mathbf{w}]\rangle \mid[\mathbf{w}] \in \mathbb{F}_{q}^{2 n} / \mathrm{V}^{\perp_{J}}\right\}$. The state of $\mathcal{A}$ is initialized as $|[\mathbf{0}]\rangle\langle[\mathbf{0}]| \otimes \rho_{\text {mix }}$ and is distributed so that the $s$-th server has $\mathcal{A}_{s}$ for $s=1,2, \ldots, \mathrm{n}$.

2) [Query] The user randomly chooses a matrix $R$ in $\mathbb{F}_{q}^{2 \mathrm{t} \times 2(\mathrm{n}-\mathrm{t}) \mathrm{f}}$ with the uniform distribution. Depending on $k$, let $E_{k}:=\left(\delta_{i, j-2(\mathrm{n}-\mathrm{t})(k-1)}\right)_{i, j} \in \mathbb{F}_{q}^{2(\mathrm{n}-\mathrm{t}) \times 2(\mathrm{n}-\mathrm{t}) \mathrm{f}}$, where $\delta_{x, y}=1$ if $x=y$ and $\delta_{x, y}=0$ if $x \neq y$. That is, $E_{k}$ is the block matrix whose $k$-th block is the identity 
matrix $I \in \mathbb{F}_{q}^{2(\mathrm{n}-\mathrm{t}) \times 2(\mathrm{n}-\mathrm{t})}$ and all other blocks are zero. Let

$$
\begin{aligned}
\mathbf{q} & =\left(\mathbf{q}_{1 X}^{\top}, \ldots, \mathbf{q}_{\mathrm{n} X}^{\top}, \mathbf{q}_{1 Z}^{\top}, \ldots, \mathbf{q}_{\mathrm{n} Z}^{\top}\right)^{\top} \\
& :=D_{1} R+D_{2} E_{k} \in \mathbb{F}_{q}^{2 \mathrm{n} \times 2(\mathrm{n}-\mathrm{t}) \mathrm{f}} .
\end{aligned}
$$

The user sends the query $\mathbf{q}_{s}=\left(\mathbf{q}_{s X}, \mathbf{q}_{s Z}\right) \in \mathbb{F}_{q}^{2(\mathrm{n}-\mathrm{t}) \mathrm{f}} \times$ $\mathbb{F}_{q}^{2(\mathrm{n}-\mathrm{t}) \mathrm{f}}$ to the $s$-th server for $s=1,2, \ldots, \mathrm{n}$.

3) [Download] For each $s=1,2, \ldots, \mathrm{n}$, the $s$-th server applies the unitary operation $\mathrm{X}\left(\mathbf{q}_{s X}^{\top} \mathbf{m}\right) \mathrm{Z}\left(\mathbf{q}_{s Z}^{\top} \mathbf{m}\right)$ to $\mathcal{A}_{s}$ and sends $\mathcal{A}_{s}$ to the user.

4) [Recovery] The user applies the PVM $\mathrm{M}^{\mathrm{V}}=\left\{P_{[\mathrm{w}]}^{\mathrm{V}} \mid\right.$ $\left.[\mathbf{w}] \in \mathbb{F}_{q}^{2 \mathrm{n}} / \mathrm{V}^{\perp_{J}}\right\}$ on $\mathcal{A}$, where $[\mathbf{w}]$ is the measurement outcome associated with $P_{[\mathbf{w}]}^{\mathrm{V}}$. The measurement outcome of the user is denoted by [ $\left.\mathbf{w}_{\text {out }}\right]$. In the expansion $\mathbf{w}_{\text {out }}=$ $\sum_{\mathbb{F}_{q}^{2(n-1)}}^{2 \mathrm{n}} c_{i} \mathbf{v}_{i}$, the user outputs $\left(c_{2 \mathrm{t}+1}, c_{2 \mathrm{t}+2}, \ldots, c_{2 \mathrm{n}}\right) \in$

\section{B. Analysis of Protocol 2}

In this section, we analyze the performance of Protocol 2

1) Costs and QPIR rate: The file size is $\mathrm{m}=\left|\mathbb{F}_{q}^{2(\mathrm{n}-\mathrm{t})}\right|=$ $q^{2(\mathrm{n}-\mathrm{t})}$, the download cost is $D\left(\Psi_{\mathrm{QPIR}}^{(\mathrm{m})}\right)=\operatorname{dim} \bigotimes_{s=1}^{\mathrm{n}} \mathcal{A}_{s}=$ $q^{\mathrm{n}}$, and the upload cost is $U\left(\Psi_{\mathrm{QPIR}}^{(\mathrm{m})}\right)=\left|\mathbb{F}_{q}^{2(\mathrm{n}-\mathrm{t}) \mathrm{f} \times 2 \mathrm{n}}\right|=$ $q^{4 \mathrm{nf}(\mathrm{n}-\mathrm{t})}$. Therefore, the QPIR rate is $R\left(\Psi_{\mathrm{QPIR}}^{(\mathrm{m})}\right)=$ $\log \mathrm{m} / \log D\left(\Psi_{\mathrm{QPIR}}^{(\mathrm{m})}\right)=2(\mathrm{n}-\mathrm{t}) / \mathrm{n}$.

2) Error probability: We show that the user obtains $\mathbf{m}_{k}$ without error. Let

$$
\begin{aligned}
\mathbf{w}^{\prime} & :=\mathbf{q}^{\top} \mathbf{m} \\
& =\left(\mathbf{q}_{1 X}^{\top} \mathbf{m}, \ldots, \mathbf{q}_{\mathrm{n} X}^{\top} \mathbf{m}, \mathbf{q}_{1 Z}^{\top} \mathbf{m}, \ldots, \mathbf{q}_{\mathrm{n} Z}^{\top} \mathbf{m}\right)^{\top} \in \mathbb{F}_{q}^{2 \mathrm{n}} .
\end{aligned}
$$

The state after the servers' encoding is

$$
\begin{aligned}
& \mathbf{W}\left(\mathbf{w}^{\prime}\right)\left(|[\mathbf{0}]\rangle\langle[\mathbf{0}]| \otimes \rho_{\text {mix }}\right) \mathbf{W}\left(\mathbf{w}^{\prime}\right)^{*} \\
& =\left|\left[\mathbf{w}^{\prime}\right]\right\rangle\left\langle\left[\mathbf{w}^{\prime}\right]\right| \otimes \rho_{\text {mix }},
\end{aligned}
$$

where the equality follows from Lemma 1 Thus, the measurement outcome $\left[\mathbf{w}_{\text {out }}\right]$ is $\left[\mathbf{w}^{\prime}\right]$. Note that we have

$$
\begin{aligned}
\mathbb{F}_{q}^{2 n} \ni \mathbf{w}^{\prime} & =\mathbf{q}^{\top} \mathbf{m} \\
& =D_{1} R \mathbf{m}+D_{2} E_{k} \mathbf{m} \\
& =D_{1} R \mathbf{m}+\sum_{i=1}^{2 \mathrm{n}-2 \mathrm{t}} m_{k, i} \mathbf{v}_{2 \mathrm{t}+i}
\end{aligned}
$$

and the first term $D_{1} R \mathbf{m}$ of (44) is a vector in $\mathrm{V}^{\perp_{J}}$, which implies

$$
\begin{aligned}
& {\left[\mathbf{w}_{\text {out }}\right]=\left[\mathbf{w}^{\prime}\right]=\mathbf{w}^{\prime}+\mathrm{V}^{\perp}} \\
& =\sum_{i=1}^{2 \mathrm{n}-2 \mathrm{t}} m_{k, i} \mathbf{v}_{2 \mathrm{t}+i}+\mathrm{V}^{\perp}=\left[\sum_{i=1}^{2 \mathrm{n}-2 \mathrm{t}} m_{k, i} \mathbf{v}_{2 \mathrm{t}+i}\right] .
\end{aligned}
$$

Thus, the user obtains $\left(c_{2 \mathrm{t}+1}, c_{2 \mathrm{t}+2}, \ldots, c_{2 \mathrm{n}}\right)=$ $\left(m_{k, 1}, \ldots, m_{k, 2(\mathrm{n}-\mathrm{t})}\right)=\mathbf{m}_{k}$.
3) Server secrecy: The protocol has perfect server secrecy because from (41), the state after the servers' encoding is $\left|\left[\mathbf{w}^{\prime}\right]\right\rangle\left\langle\left[\mathbf{w}^{\prime}\right]\right| \otimes \rho_{\text {mix }}$, which is independent of the non-retrieved files.

Remark 7. The server secrecy is not perfect if the prior entangled state is $|[\mathbf{0}]\rangle\langle[\mathbf{0}]| \otimes \rho$ with non-completely mixed state $\rho$. As remarked in Remark 6 , if $|[\mathbf{0}]\rangle\langle[\mathbf{0}]| \otimes \rho$ is the initial entangled state, the state $\rho$ may be changed depending on the servers' operation $\mathbf{W}\left(\mathbf{w}^{\prime}\right)$, i.e.,

$$
\mathbf{W}\left(\mathbf{w}^{\prime}\right)(|[\mathbf{0}]\rangle\langle[\mathbf{0}]| \otimes \rho) \mathbf{W}\left(\mathbf{w}^{\prime}\right)^{*}=\left|\left[\mathbf{w}^{\prime}\right]\right\rangle\left\langle\left[\mathbf{w}^{\prime}\right]\right| \otimes \rho_{\mathbf{w}^{\prime}}^{\prime}
$$

for some state $\rho_{\mathbf{w}^{\prime}}^{\prime}$. Thus, the user may obtain some information of $\mathbf{w}^{\prime}$ from the state $\rho_{\mathbf{w}^{\prime}}^{\prime}$, i.e., some information of the non-targeted files is leaked.

4) User secrecy: To discuss the user secrecy of Protocol 2. we introduce the following notations. We denote $\mathbf{v}_{i}=\left(v_{1, i}, \ldots, v_{2 \mathrm{n}, i}\right)^{\top} \in \mathbb{F}_{q}^{2 \mathrm{n}}$ for $i=1, \ldots, 2 \mathrm{n}$. For any permutation $\pi$ in $\mathrm{S}_{\mathrm{n}}$, we denote

$$
\begin{aligned}
\mathbf{v}_{i, \pi} & :=\left(\begin{array}{c}
v_{\pi(1), i} \\
\vdots \\
v_{\pi(\mathrm{t}), i} \\
v_{\mathrm{n}+\pi(1), i} \\
\vdots \\
v_{\mathrm{n}+\pi(\mathrm{t}), i}
\end{array}\right) \in \mathbb{F}_{q}^{2 \mathrm{t}}, \\
D_{1, \pi} & :=\left(\mathbf{v}_{1, \pi}, \ldots, \mathbf{v}_{2 \mathrm{t}, \pi}\right) \in \mathbb{F}_{q}^{2 \mathrm{t} \times 2 \mathrm{t}}, \\
D_{2, \pi} & :=\left(\mathbf{v}_{2 \mathrm{t}+1, \pi}, \ldots, \mathbf{v}_{2 \mathrm{n}, \pi}\right) \in \mathbb{F}_{q}^{2 \mathrm{t} \times 2(\mathrm{n}-\mathrm{t})} .
\end{aligned}
$$

The user t-secrecy is proved as follows. Let $\pi$ be an arbitrary permutation in $\mathrm{S}_{\mathrm{n}}$. The queries to the $\pi(1)$-th server, $\ldots, \pi(\mathrm{t})$ th server are written as

$$
\begin{aligned}
& \left(\mathbf{q}_{\pi(1) X}, \ldots, \mathbf{q}_{\pi(\mathrm{t}) X}, \mathbf{q}_{\pi(1) Z}, \ldots, \mathbf{q}_{\pi(\mathrm{t}) Z}\right)^{\top} \\
& =D_{1, \pi} R+D_{2, \pi} E_{k} \in \mathbb{F}_{q}^{2 \mathrm{t} \times 2(\mathrm{n}-\mathrm{t}) \mathrm{f}} .
\end{aligned}
$$

Since condition (a) of Lemma 2 implies rank $D_{1, \pi}=2 \mathrm{t}$, i.e., $D_{1, \pi}$ is invertible, when $R$ is uniformly random in $\mathbb{F}_{q}^{2 \mathrm{t} \times 2(\mathrm{n}-\mathrm{t}) \mathrm{f}}$, the distribution of $\left(\mathbf{q}_{\pi(1) X}, \ldots, \mathbf{q}_{\pi(\mathrm{t}) X}, \mathbf{q}_{\pi(1) Z}, \ldots, \mathbf{q}_{\pi(\mathrm{t}) Z}\right)^{\top}$ is the uniform distribution on $\mathbb{F}_{q}^{2 \mathrm{t} \times 2(\mathrm{n}-\mathrm{t}) \mathrm{f}}$. Therefore, the colluding servers obtain no information of the target file index $k$ since the matrix $R$ is unknown to the colluding servers and is uniformly random in $\mathbb{F}_{q}^{2 \mathrm{t} \times 2(\mathrm{n}-\mathrm{t}) \mathrm{f}}$.

\section{Converse Bounds}

The converse bounds of Theorem 1 are written for any $\alpha \in$ $[0,1)$ and any $\beta, \gamma, \theta \in[0, \infty)$ as

$$
\begin{aligned}
C_{\text {asymp }, \mathrm{t}}^{\alpha, \beta, \gamma}, \boldsymbol{} \leq 1 & \text { if } 1 \leq \mathrm{t} \leq \frac{\mathrm{n}}{2}, \\
C_{\text {exact, }}^{\alpha, 0,0, \theta} \leq \frac{2(\mathrm{n}-\mathrm{t})}{\mathrm{n}} & \text { if } \frac{\mathrm{n}}{2}<\mathrm{t}<\mathrm{n}, \\
C_{\text {asymp, }}^{0, \beta, 0, \theta} \leq \frac{2(\mathrm{n}-\mathrm{t})}{\mathrm{n}} & \text { if } \frac{\mathrm{n}}{2}<\mathrm{t}<\mathrm{n} .
\end{aligned}
$$

The bounds (50) and (51) are proved similar to the converse proofs of [27] and [28], respectively. Therefore, we give the details of the two proofs in Appendix $\mathrm{F}$ and in this section, we give the proof of (52). 


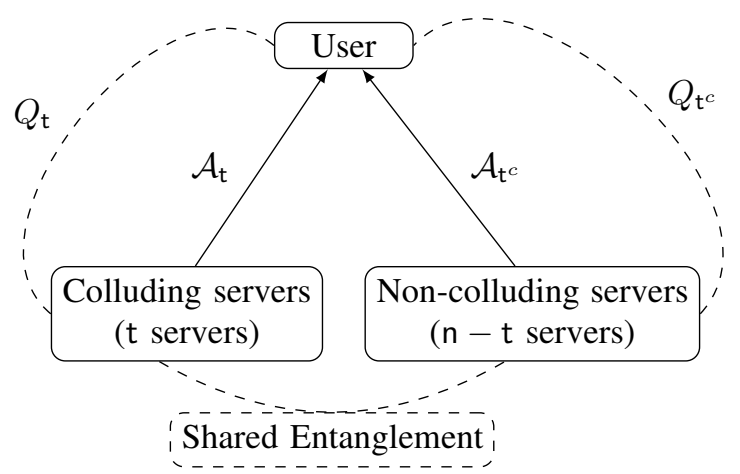

(a) Downloading step of QPIR protocol. The user shares $Q_{\mathrm{t}}$ with colluding servers and $Q_{\mathrm{t}^{c}}$ with non-colluding servers.

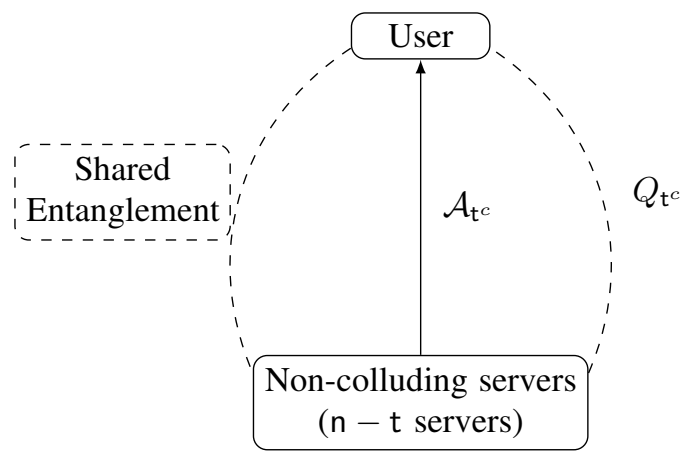

(b) Entanglement-assisted communication of classical message with shared randomness $Q_{\mathrm{t}}$. Note that the user know $Q$ but not which query $Q_{\mathrm{t}^{c}}$ the non-colluding servers contain.

Fig. 4: Proof idea of converse bound. By the secrecy conditions, the downloading step (a) can be considered as (b). Here, we denote $\mathcal{A}_{\mathrm{t}}:=\bigotimes_{s=1}^{\mathrm{t}} \mathcal{A}_{s}, \mathcal{A}_{\mathrm{t}^{c}}:=\bigotimes_{s=\mathrm{t}+1}^{\mathrm{n}} \mathcal{A}_{s}$, $Q_{\mathrm{t}}:=\left(Q_{1}, \ldots, Q_{\mathrm{t}}\right)$, and $Q_{\mathrm{t}^{c}}:=\left(Q_{\mathrm{t}+1}, \ldots, Q_{\mathrm{n}}\right)$ for $\pi \in \mathrm{S}$.

The proof idea of the converse bounds $(51),(52)$ is illustrated in Fig. 4 and is explained as follows. By the secrecy conditions, the state on $\bigotimes_{s=1}^{\mathrm{t}} \mathcal{A}_{\pi(s)}$ from the colluding servers is independent of the file information, which will be precisely stated in Lemma 4 With this fact, the state on $\bigotimes_{s=1}^{\mathrm{t}} \mathcal{A}_{\pi(s)}$ can be considered as shared entanglement between the user and the non-colluding servers. That is, the downloading step of the protocol (Fig. 4-(a)) can be considered as the entanglement-assisted communication of a classical message (Fig. 4-(b)). Since the capacity of the entanglement-assisted classical communication [46] for the identity channel is two times the dimension of the transmitted quantum systems, the PIR capacity is upper bounded by $2(n-t) / n$ and the tightness of this bound is guaranteed by the QPIR protocol in Section $\mathrm{V}$. The bound (50) is satisfied because the retrieved file size cannot exceed the dimension of downloaded quantum systems, but we give the formal proof in Appendix $\mathrm{F}$

\section{A. Proof of converse bound (52)}

First, we prepare the following lemma for the converse bound (52).

Lemma 3. Let $\Psi_{\mathrm{QPIR}}^{(\mathrm{m})}$ be a t-private QPIR protocol such that

$$
\begin{aligned}
& S_{\text {serv }}\left(\Psi_{\mathrm{QPIR}}^{(\mathrm{m})}\right) \leq \beta, \\
& S_{\mathrm{user}}^{(\mathrm{t})}\left(\Psi_{\mathrm{QPIR}}^{(\mathrm{m})}\right) \leq \gamma, \\
& P_{\mathrm{err}}\left(\Psi_{\mathrm{QPIR}}^{(\mathrm{m})}\right) \leq \min \{1 / 2,1-10 \sqrt{2 \mathrm{f} \gamma}\} .
\end{aligned}
$$

Then, the protocol $\Psi_{\mathrm{QPIR}}^{(\mathrm{m})}$ satisfies

$$
\log \mathrm{m} \leq \frac{2(\mathrm{n}-\mathrm{t}) \log \mathrm{d}+f\left(P_{\mathrm{err}}\left(\Psi_{\mathrm{QPIR}}^{(\mathrm{m})}\right), \beta, \gamma, \mathrm{f}\right)}{1-P_{\mathrm{err}}\left(\Psi_{\mathrm{QPIR}}^{(\mathrm{m})}\right)-10 \sqrt{2 \mathrm{f} \gamma}},
$$

where $f(\alpha, \beta, \gamma, \mathrm{f}):=\beta+\eta_{0}(2 \sqrt{2 \mathrm{f} \gamma})+2 h_{2}(2 \sqrt{2 \mathrm{f} \gamma})+h_{2}(\alpha)$, $h_{2}(x)$ is the binary entropy function $h_{2}(x):=-x \log x-(1-$ $x) \log (1-x)$, and

$$
\eta_{0}(x):= \begin{cases}1 / e & \text { if } 1 / e<x, \\ -x \log x & \text { if } 0<x<1 / e .\end{cases}
$$

Lemma 3 will be proved in Section VI-B

Now, we prove the converse bound $(\underline{52})$. Let $\left\{\Psi_{\mathrm{QPIR}}^{\left(\mathrm{m}_{\ell}\right)}\right\}_{\ell=1}^{\infty}$ be any sequence of QPIR protocols such that

$\left(\alpha_{\ell}, \beta_{\ell}, \gamma_{\ell}\right):=\left(P_{\mathrm{err}}\left(\Psi_{\mathrm{QPIR}}^{\left(\mathrm{m}_{\ell}\right)}\right), S_{\mathrm{serv}}\left(\Psi_{\mathrm{QPIR}}^{\left(\mathrm{m}_{\ell}\right)}\right), S_{\mathrm{user}}^{(\mathrm{t})}\left(\Psi_{\mathrm{QPIR}}^{(\mathrm{m})} \mathrm{m}_{\ell}\right)\right)$

satisfies

$$
\begin{aligned}
& \limsup _{\ell \rightarrow \infty} \alpha_{\ell}=0, \\
& \limsup _{\ell \rightarrow \infty} \gamma_{\ell}=0, \\
& \limsup _{\ell \rightarrow \infty} \beta_{\ell}=\beta .
\end{aligned}
$$

Let $\mathrm{d}_{\ell}$ be the dimension of $\mathcal{A}_{s}(\forall s \in\{1, \ldots, \mathrm{n}\})$ for the protocol $\Psi_{\mathrm{QPIR}}^{\left(\mathrm{m}_{\ell}\right)}$. Then, for any sufficiently large $\ell$ such that $\alpha_{\ell} \leq \min \left\{1 / 2,1-10 \sqrt{2 \mathrm{f} \gamma_{\ell}}\right\}$, Lemma 3 gives

$$
\log \mathrm{m}_{\ell} \leq \frac{2(\mathrm{n}-\mathrm{t}) \log \mathrm{d}_{\ell}+f\left(\alpha_{\ell}, \beta_{\ell}, \gamma_{\ell}, \mathrm{f}\right)}{1-\alpha_{\ell}-10 \sqrt{2 \mathrm{f} \gamma_{\ell}}} .
$$

Hence, the asymptotic QPIR rate satisfies

$$
\begin{aligned}
\lim _{\ell \rightarrow \infty} R\left(\Psi_{\mathrm{QPIR}}^{\left(\mathrm{m}_{\ell}\right)}\right) & =\lim _{\ell \rightarrow \infty} \frac{\log \mathrm{m}_{\ell}}{\mathrm{n} \log \mathrm{d}_{\ell}} \\
& \leq \lim _{\ell \rightarrow \infty} \frac{2(\mathrm{n}-\mathrm{t}) \log \mathrm{d}_{\ell}+f\left(\alpha_{\ell}, \beta_{\ell}, \gamma_{\ell}, \mathrm{f}\right)}{\left(1-\alpha_{\ell}-10 \sqrt{2 \mathrm{f} \gamma_{\ell}}\right) \mathrm{n} \log \mathrm{d}_{\ell}} \\
& =\frac{2(\mathrm{n}-\mathrm{t})}{\mathrm{n}}
\end{aligned}
$$

where the last equality follows from the relation $\left(\alpha_{\ell}, \beta_{\ell}, \gamma_{\ell}, \mathrm{d}_{\ell}\right) \rightarrow(0, \beta, 0, \infty)$ as $\ell \rightarrow \infty$. Thus, we obtain the converse bound (52). 


\section{B. Proof of Lemma 3}

In this section, we prove Lemma 3 Without losing generality, we fix an arbitrary $\pi \in \mathrm{S}_{\mathrm{n}}$ in the following proofs. We use the notation

$$
\begin{aligned}
Q_{\mathrm{t}} & :=\left(Q_{\pi(1)}, \ldots, Q_{\pi(\mathrm{t})}\right), \\
Q_{\mathrm{t}^{c}} & :=\left(Q_{\pi(\mathrm{t}+1)}, \ldots, Q_{\pi(\mathrm{n})}\right), \\
\mathcal{A}_{\mathrm{t}} & :=\bigotimes_{s=1}^{\mathrm{t}} \mathcal{A}_{\pi(s)}, \\
\mathcal{A}_{\mathrm{t}^{c}} & :=\bigotimes_{s=\mathrm{t}+1}^{\mathrm{n}} \mathcal{A}_{\pi(s)} .
\end{aligned}
$$

We also denote by $\rho\left(M, Q_{\mathrm{t}}\right)$ the state on $\mathcal{A}_{\mathrm{t}}$ of the t-colluding servers after the servers' encoding.

To show Lemma 3, we prepare the following lemma.

Lemma 4. If a QPIR protocol $\Psi_{\mathrm{QPIR}}^{(\mathrm{m})}$ satisfies $S_{\mathrm{serv}}\left(\Psi_{\mathrm{QPIR}}^{(\mathrm{m})}\right) \leq \beta$ and $S_{\mathrm{user}}^{(\mathrm{t})}\left(\Psi_{\mathrm{QPIR}}^{(\mathrm{m})}\right) \leq \gamma$, then for any $k \in\{1, \ldots, \mathrm{f}\}$, we have the relation

$$
I\left(M_{k} ; \mathcal{A}_{\mathrm{t}} \mid Q_{\mathrm{t}}, K=k\right)_{\rho\left(M, Q_{\mathrm{t}}\right)} \leq \beta+g(\mathrm{~m}, \gamma)
$$

after the servers' encoding, where

$$
g(\mathrm{~m}, \gamma):=10 \sqrt{2 \mathrm{f} \gamma} \log \mathrm{m}+\eta_{0}(2 \sqrt{2 \mathrm{f} \gamma})+2 h_{2}(2 \sqrt{2 \mathrm{f} \gamma}) .
$$

In particular, when $\gamma=0$, we have $I\left(M_{k} ; \mathcal{A}_{\mathrm{t}} \mid Q_{\mathrm{t}}, K=\right.$ $k)_{\rho\left(M, Q_{\mathrm{t}}\right)} \leq \beta$ for any $k \in\{1, \ldots, \mathrm{f}\}$.

Lemma 4 is proved in Appendix [E] We also prepare the following proposition for the classical and quantum relative entropies defined in Section [II]

Proposition 3 ( [41, (3.18)] ). The information-processing inequality for quantum relative entropy with respect to the two-valued measurement $\{Y, I-Y\}$ is written as

$$
\begin{aligned}
D(\rho \| \sigma) & \geq D\left(P_{\rho} \| P_{\sigma}\right) \\
& =-h_{2}\left(P_{\rho}(1)\right)-P_{\rho}(1) \log P_{\sigma}(1)-P_{\rho}(2) \log P_{\sigma}(2),
\end{aligned}
$$

where $P_{\rho}=\left\{P_{\rho}(1), P_{\rho}(2)\right\}=\{\operatorname{Tr} \rho Y, \operatorname{Tr} \rho(I-Y)\}, P_{\sigma}=$ $\left\{P_{\sigma}(1), P_{\sigma}(2)\right\}=\{\operatorname{Tr} \sigma Y, \operatorname{Tr} \sigma(I-Y)\}$.

Now, we prove Lemma 3 by four steps.

Step 1: First, we prepare the following notation. Fix $K=k$ arbitrarily. Let $\rho\left(m_{k}, m_{k}^{c}, q\right)$ be the quantum state on the composite system $\bigotimes_{s=1}^{n} \mathcal{A}_{s}$, where $m_{k}$ is the file to be retrieved, $m_{k}^{c}$ is the collection of non-retrieved $f-1$ files, and $q$ is the collection of queries. Note that in view of Fig. 4. (b), the target file $m_{k}$ corresponds to the classical message and the query $q$ determines the decoding algorithm, but $m_{k}^{c}$ is redundant information since it is independent of $m_{k}$ and $q$. Therefore, in the following, we only consider the states averaged with respect to $m_{k}^{c}$ as

$$
\begin{aligned}
\rho\left(m_{k}, q\right) & =\frac{1}{\mathrm{~m}^{\mathrm{f}-1}} \sum_{m_{k}^{c}} \rho\left(m_{k}, m_{k}^{c}, q\right), \\
\rho(q) & =\frac{1}{\mathrm{~m}} \sum_{m_{k}=1}^{\mathrm{m}} \rho\left(m_{k}, q\right) .
\end{aligned}
$$

Considering the entire system $\mathcal{A}$ as a bipartite system $\mathcal{A}_{\mathbf{t}} \otimes \mathcal{A}_{\mathbf{t}^{c}}$, let $\rho\left(m_{k}, q_{\mathrm{t}}\right)$ and $\rho\left(q_{\mathrm{t}}\right)$ be the reduced densities of $\rho\left(m_{k}, q\right)$ and $\rho(q)$ on $\mathcal{A}_{\mathrm{t}}$, respectively. Remind that the decoding POVM is denoted by $\left\{Y_{k, q}(w)\right\}_{w \in\{1, \ldots, \mathrm{m}\}}$, which depends on $k$ and $q$. Then, we define the states

$$
\begin{aligned}
\tilde{\rho}\left(M_{k} \mid q\right) & :=\sum_{m_{k}=1}^{\mathrm{m}} \frac{1}{\mathrm{~m}}\left|m_{k}\right\rangle\left\langle m_{k}\right| \otimes \rho\left(m_{k}, q\right), \\
\tilde{\rho}\left(q_{\mathrm{t}}\right) & :=\sum_{m_{k}=1}^{\mathrm{m}} \frac{1}{\mathrm{~m}}\left|m_{k}\right\rangle\left\langle m_{k}\right| \otimes \rho\left(q_{\mathrm{t}}\right) \otimes I / \mathrm{d}^{\mathrm{n}-\mathrm{t}} \\
& =\frac{I}{\mathrm{~m}} \otimes \rho\left(q_{\mathrm{t}}\right) \otimes \frac{I}{\mathrm{~d}^{\mathrm{n}-\mathrm{t}}}
\end{aligned}
$$

and the operator $Y_{k, q}:=\sum_{m_{k}=1}^{\mathrm{m}}\left|m_{k}\right\rangle\left\langle m_{k}\right| \otimes Y_{k, q}\left(m_{k}\right)$.

Step 2: In this step, we derive

$$
\begin{aligned}
& \left(1-P_{\mathrm{err}, k}\left(\Psi_{\mathrm{QPIR}}^{(\mathrm{m})}\right)\right) \log \mathrm{m} \\
& \leq \mathbb{E}_{Q} D\left(\tilde{\rho}\left(M_{k} \mid Q\right) \| \tilde{\rho}\left(Q_{\mathrm{t}}\right)\right)+h_{2}\left(P_{\mathrm{err}, k}\left(\Psi_{\mathrm{QPIR}}^{(\mathrm{m})}\right)\right)
\end{aligned}
$$

for any $k \in\{1, \ldots, f\}$, where

$$
P_{\mathrm{err}, k}\left(\Psi_{\mathrm{QPIR}}^{(\mathrm{m})}\right):=\operatorname{Pr}_{W, M_{k}}\left[W \neq M_{k} \mid K=k\right] .
$$

We prove (70) by applying Proposition 3 to the states $\tilde{\rho}\left(M_{k} \mid q\right)$ and $\tilde{\rho}\left(q_{\mathrm{t}}\right)$ and the measurement $\left\{Y_{k, q}, I-Y_{k, q}\right\}$ as follows. In this application of Proposition 3 , the distributions $P_{\tilde{\rho}\left(M_{k} \mid q\right)}$ and $P_{\tilde{\rho}\left(q_{\mathrm{t}}\right)}$ are written as

$$
\begin{aligned}
P_{\tilde{\rho}\left(M_{k} \mid q\right)} & =\left\{P_{\tilde{\rho}\left(M_{k} \mid q\right)}(1), P_{\tilde{\rho}\left(M_{k} \mid q\right)}(2)\right\} \\
& =\left\{P_{\mathrm{err}, k, q}\left(\Psi_{\mathrm{QPIR}}^{(\mathrm{m})}\right), 1-P_{\mathrm{err}, k, q}\left(\Psi_{\mathrm{QPIR}}^{(\mathrm{m})}\right)\right\}, \\
P_{\tilde{\rho}\left(q_{\mathrm{t}}\right)} & =\left\{P_{\tilde{\rho}\left(q_{\mathrm{t}}\right)}(1), P_{\tilde{\rho}\left(q_{\mathrm{t}}\right)}(2)\right\}=\left\{\frac{1}{\mathrm{~m}}, 1-\frac{1}{\mathrm{~m}}\right\},
\end{aligned}
$$

where

$$
P_{\mathrm{err}, k, q}\left(\Psi_{\mathrm{QPIR}}^{(\mathrm{m})}\right):=\operatorname{Pr}_{W, M_{k}}\left[W \neq M_{k} \mid K=k, Q=q\right] .
$$

Thus, applying Proposition 3 , we have

$$
\begin{aligned}
& \left(1-P_{\mathrm{err}, k, q}\left(\Psi_{\mathrm{QPIR}}^{(\mathrm{m})}\right)\right) \log \mathrm{m} \\
& =-P_{\tilde{\rho}\left(M_{k} \mid q\right)}(2) \log P_{\tilde{\rho}\left(q_{\mathrm{t}}\right.}(2) \\
& \stackrel{(a)}{\leq}-P_{\tilde{\rho}\left(M_{k} \mid q\right)}(2) \log P_{\tilde{\rho}\left(q_{\mathrm{t}}\right.}(2)-P_{\tilde{\rho}\left(M_{k} \mid q\right)}(1) \log P_{\tilde{\rho}\left(q_{\mathrm{t}}\right)}(1) \\
& \leq D\left(\tilde{\rho}\left(M_{k} \mid q\right) \| \tilde{\rho}\left(q_{\mathrm{t}}\right)\right)+h_{2}\left(P_{\tilde{\rho}\left(M_{k} \mid q\right)}(1)\right) \\
& =D\left(\tilde{\rho}\left(M_{k} \mid q\right) \| \tilde{\rho}\left(q_{\mathrm{t}}\right)\right)+h_{2}\left(P_{\mathrm{err}, k, q}\left(\Psi_{\mathrm{QPIR}}^{(\mathrm{m})}\right)\right)
\end{aligned}
$$

where $(a)$ is from $P_{\tilde{\rho}\left(M_{k} \mid q\right)}(1) \log P_{\tilde{\rho}\left(q_{\mathrm{t}}\right)}(1) \leq 0$. Taking the expectation with respect to $Q$ and using the concavity of $h_{2}$, we have

$$
\begin{aligned}
& \left(1-P_{\mathrm{err}, k}\left(\Psi_{\mathrm{QPIR}}^{(\mathrm{m})}\right)\right) \log \mathrm{m} \\
& =\mathbb{E}_{Q}\left(1-P_{\mathrm{err}, k, Q}\left(\Psi_{\mathrm{QPIR}}^{(\mathrm{m})}\right)\right) \log \mathrm{m} \\
& \leq \mathbb{E}_{Q} D\left(\tilde{\rho}\left(M_{k} \mid Q\right) \| \tilde{\rho}\left(Q_{\mathrm{t}}\right)\right)+\mathbb{E}_{Q} h_{2}\left(P_{\mathrm{err}, k, Q}\left(\Psi_{\mathrm{QPIR}}^{(\mathrm{m})}\right)\right) \\
& \leq \mathbb{E}_{Q} D\left(\tilde{\rho}\left(M_{k} \mid Q\right) \| \tilde{\rho}\left(Q_{\mathrm{t}}\right)\right)+h_{2}\left(P_{\mathrm{err}, k}\left(\Psi_{\mathrm{QPIR}}^{(\mathrm{m})}\right)\right)
\end{aligned}
$$

which is the desired inequality (70). 
Step 3: Next, we derive

$$
\begin{aligned}
& \mathbb{E}_{Q} D\left(\tilde{\rho}\left(M_{k} \mid Q\right) \| \tilde{\rho}\left(Q_{\mathrm{t}}\right)\right) \\
& \quad \leq 2(\mathrm{n}-\mathrm{t}) \log \mathrm{d}+I\left(M_{k} ; \mathcal{A}_{\mathrm{t}} \mid Q_{\mathrm{t}}, K=k\right)_{\rho\left(M, Q_{\mathrm{t}}\right)}
\end{aligned}
$$

as follows. The inequality (76) is derived by

$$
\begin{aligned}
& \mathbb{E}_{Q} D\left(\tilde{\rho}\left(M_{k} \mid Q\right) \| \tilde{\rho}\left(Q_{\mathrm{t}}\right)\right) \\
& =\mathbb{E}_{Q} \frac{1}{\mathrm{~m}} \sum_{m_{k}=1}^{\mathrm{m}} D\left(\rho\left(m_{k}, Q\right) \| \rho\left(Q_{\mathrm{t}}\right) \otimes \frac{I}{\mathrm{~d}^{\mathrm{n}-\mathrm{t}}}\right) \\
& \stackrel{(b)}{=} \mathbb{E}_{Q} \frac{1}{\mathrm{~m}} \sum_{m_{k}=1}^{\mathrm{m}} D\left(\rho\left(m_{k}, Q\right) \| \rho\left(m_{k}, Q_{\mathrm{t}}\right) \otimes \frac{I}{\mathrm{~d}^{\mathrm{n}-\mathrm{t}}}\right) \\
& \quad+\mathbb{E}_{Q} \frac{1}{\mathrm{~m}} \sum_{m_{k}=1}^{\mathrm{m}} D\left(\rho\left(m_{k}, Q_{\mathrm{t}}\right) \| \rho\left(Q_{\mathrm{t}}\right)\right) \\
& =\mathbb{E}_{Q} \frac{1}{\mathrm{~m}} \sum_{m_{k}=1}^{\mathrm{m}} D\left(\rho\left(m_{k}, Q\right) \| \rho\left(m_{k}, Q_{\mathrm{t}}\right) \otimes \frac{I}{\mathrm{~d}^{\mathrm{n}-\mathrm{t}}}\right) \\
& \quad+I\left(M_{k} ; \mathcal{A}_{\mathrm{t}} \mid Q_{\mathrm{t}}, K=k\right)_{\rho\left(M, Q_{\mathrm{t}}\right)} \\
& \stackrel{(c)}{\leq} 2(\mathrm{n}-\mathrm{t}) \log \mathrm{d}+I\left(M_{k} ; \mathcal{A}_{\mathrm{t}} \mid Q_{\mathrm{t}}, K=k\right)_{\rho\left(M, Q_{\mathrm{t}}\right)} .
\end{aligned}
$$

The equation $(b)$ can be shown as follows.

$$
\begin{aligned}
D & \left(\rho\left(m_{k}, Q\right) \| \rho\left(Q_{\mathrm{t}}\right) \otimes \frac{I}{\mathrm{~d}^{\mathrm{n}-\mathrm{t}}}\right) \\
= & \operatorname{Tr} \rho\left(m_{k}, Q\right)\left(\log \rho\left(m_{k}, Q\right)-\log \left(\rho\left(Q_{\mathrm{t}}\right) \otimes \frac{I}{\mathrm{~d}^{\mathrm{n}-\mathrm{t}}}\right)\right) \\
= & \operatorname{Tr} \rho\left(m_{k}, Q\right)\left(\log \rho\left(m_{k}, Q\right)-\log \left(\rho\left(m_{k}, Q_{\mathrm{t}}\right) \otimes \frac{I}{\mathrm{~d}^{\mathrm{n}-\mathrm{t}}}\right)\right. \\
& \left.+\log \left(\rho\left(m_{k}, Q_{\mathrm{t}}\right) \otimes \frac{I}{\mathrm{~d}^{\mathrm{n}-\mathrm{t}}}\right)-\log \left(\rho\left(Q_{\mathrm{t}}\right) \otimes \frac{I}{\mathrm{~d}^{\mathrm{n}-\mathrm{t}}}\right)\right) \\
= & D\left(\rho\left(m_{k}, Q\right) \| \rho\left(m_{k}, Q_{\mathrm{t}}\right) \otimes \frac{I}{\mathrm{~d}^{\mathrm{n}-\mathrm{t}}}\right) \\
& +D\left(\rho\left(m_{k}, Q_{\mathrm{t}}\right) \| \rho\left(Q_{\mathrm{t}}\right)\right) .
\end{aligned}
$$

The inequality $(c)$ can be shown as follows. We diagonalize $\rho\left(m_{k}, Q\right)=\sum_{i} p_{i}\left|\phi_{i}\right\rangle\left\langle\phi_{i}\right|$ and denote by $\rho_{i}$ the reduced density of $\left|\phi_{i}\right\rangle\left\langle\phi_{i}\right|$ on $\mathcal{A}_{\mathrm{t}}$. Then,

$$
\begin{aligned}
& D\left(\rho\left(m_{k}, Q\right) \| \rho\left(m_{k}, Q_{\mathrm{t}}\right) \otimes \frac{I}{\mathrm{~d}^{\mathrm{n}-\mathrm{t}}}\right) \\
& \leq \sum_{i} p_{i} D\left(\left|\phi_{i}\right\rangle\left\langle\phi_{i}\right| \| \rho_{i} \otimes \frac{I}{\mathrm{~d}^{\mathrm{n}-\mathrm{t}}}\right) \\
& =\log \mathrm{d}^{\mathrm{n}-\mathrm{t}}+\sum_{i} p_{i} H\left(\rho_{i}\right) \leq 2 \log \mathrm{d}^{\mathrm{n}-\mathrm{t}},
\end{aligned}
$$

where the last inequality is proved from $H\left(\rho_{i}\right)=H\left(\rho_{i}^{\prime}\right) \leq$ $\log \mathrm{d}^{\mathrm{n}-\mathrm{t}}$ for the reduced density $\rho_{i}^{\prime}$ of $\left|\phi_{i}\right\rangle\left\langle\phi_{i}\right|$ on $\mathcal{A}_{\mathrm{t}^{c}}$.

Step 4: Lastly, we prove (56) of Lemma 3 Combining (70), (76), and Lemma 4, we have

$$
\begin{aligned}
(1 & \left.-P_{\mathrm{err}, k}\left(\Psi_{\mathrm{QPIR}}^{(\mathrm{m})}\right)\right) \log \mathrm{m} \\
\leq & 2(\mathrm{n}-\mathrm{t}) \log \mathrm{d}+I\left(M_{k} ; \mathcal{A}_{\mathrm{t}} \mid Q_{\mathrm{t}}, K=k\right)_{\rho\left(M, Q_{\mathrm{t}}\right)} \\
& +h_{2}\left(P_{\mathrm{err}, k}\left(\Psi_{\mathrm{QPIR}}^{(\mathrm{m})}\right)\right) \\
\leq & 2(\mathrm{n}-\mathrm{t}) \log \mathrm{d}+\beta+g(\mathrm{~m}, \gamma)+h_{2}\left(P_{\mathrm{err}, k}\left(\Psi_{\mathrm{QPIR}}^{(\mathrm{m})}\right)\right) \\
= & 2(\mathrm{n}-\mathrm{t}) \log \mathrm{d}+\beta+10 \sqrt{2 \mathrm{f} \gamma} \log \mathrm{m}+\eta_{0}(2 \sqrt{2 \mathrm{f} \gamma})
\end{aligned}
$$

$$
\begin{aligned}
& +2 h_{2}(2 \sqrt{2 \mathrm{f} \gamma})+h_{2}\left(P_{\mathrm{err}, k}\left(\Psi_{\mathrm{QPIR}}^{(\mathrm{m})}\right)\right) \\
= & 2(\mathrm{n}-\mathrm{t}) \log \mathrm{d}+f\left(P_{\mathrm{err}, k}\left(\Psi_{\mathrm{QPIR}}^{(\mathrm{m})}\right), \beta, \gamma, \mathrm{f}\right)
\end{aligned}
$$

Then, rewriting the above inequality, we obtain Lemma 3 as

$$
\begin{aligned}
\log \mathrm{m} & \leq \frac{2(\mathrm{n}-\mathrm{t}) \log \mathrm{d}+f\left(P_{\mathrm{err}, k}\left(\Psi_{\mathrm{QPIR}}^{(\mathrm{m})}\right), \beta, \gamma, \mathrm{f}\right)}{1-P_{\mathrm{err}, k}\left(\Psi_{\mathrm{QPIR}}^{(\mathrm{m})}\right)-10 \sqrt{2 \mathrm{f} \gamma}} \\
& \stackrel{(d)}{\leq} \frac{2(\mathrm{n}-\mathrm{t}) \log \mathrm{d}+f\left(P_{\mathrm{err}}\left(\Psi_{\mathrm{QPIR}}^{(\mathrm{m})}\right), \beta, \gamma, \mathrm{f}\right)}{1-P_{\mathrm{err}}\left(\Psi_{\mathrm{QPIR}}^{(\mathrm{m})}\right)-10 \sqrt{2 \mathrm{f} \gamma}},
\end{aligned}
$$

where $(d)$ holds because $P_{\mathrm{err}, k}\left(\Psi_{\mathrm{QPIR}}^{(\mathrm{m})}\right) \leq P_{\mathrm{err}}\left(\Psi_{\mathrm{QPIR}}^{(\mathrm{m})}\right)<$ $1 / 2$ and the binary entropy $h_{2}(p)$ is inceasing for $p \leq 1 / 2$.

Remark 8. In Step 2, we condition on $Q=q$ and then take expectation with respect to $Q$. The reason why we condition on $Q=q$ is that the states and the decoder are determined depending on the value of $Q$. Thus, to derive (72) which relates the error probability and Proposition 3 , we need to condition on $Q=q$. On the other hand, we need to take expectation on $Q$ in (75) because we need to recover $Q_{\mathrm{t}}$ as a random variable to apply the user secrecy condition $I\left(K ; Q_{\mathrm{t}}\right) \leq \gamma$. To be precise, we use the user secrecy condition $I\left(K ; Q_{\mathrm{t}}\right) \leq \gamma$ in the proof of Lemma 4 and we apply Lemma 4 in Step 4.

\section{CONCLUSION}

We have discussed symmetric t-private quantum private information retrieval. We have considered two secrecy conditions, the user t-secrecy and the server secrecy. The user $\mathrm{t}$-secrecy is the secrecy in which any collection of $\mathrm{t}$ queries contains no information of the user's request, and the server secrecy is the secrecy in which the user obtains no information of other files than the target file. We have derived two capacities of t-private QPIR, the exact and asymptotic security-constrained capacities. When the number of colluding servers $t$ is less than or equal to half of the number of servers $\mathrm{n}$, the capacities are exactly 1 whether considering the security conditions or not and if $t>n / 2$, the capacities are $2(n-t) / n$. For the proof of the capacities, we have constructed a symmetric t-private QPIR protocol with perfect security conditions by the stabilizer formalism. We have also derived the converse bounds, which complete the optimality of our protocol.

There are many open problems related to t-private QPIR. t-Private QPIR with adversarial models needs to be discussed since we only considered the most trivial security model in which the user and the servers follow the protocol and do not deviate from the protocol. t-Private QPIR capacity should also be discussed for the multi-round case and for the model without shared entanglement.

\section{ACKNOWLEDGMENTS}

The authors are grateful to Yuuya Yoshida for providing a concise proof of Proposition 4 and helpful comments. 


\section{APPENDIX A}

\section{QPIR Capacity with Average Security Measures}

In Section III-B, we defined the security measures as the worst-case definition. In this appendix, we show that the capacity does not changes even if we change the definition of the security measures as for the average case.

Define the average security measures as

$$
\begin{aligned}
\tilde{P}_{\mathrm{err}}\left(\Psi_{\mathrm{QPIR}}^{(\mathrm{m})}\right) & :=\operatorname{Pr}_{W}\left[W \neq M_{K} \mid M Q K\right] \\
\tilde{S}_{\mathrm{serv}}\left(\Psi_{\mathrm{QPIR}}^{(\mathrm{m})}\right) & :=I\left(M_{K}^{c} ; \mathcal{A} \mid Q K\right)_{\rho(M, Q)} \\
\tilde{S}_{\mathrm{user}}^{(\mathrm{t})}\left(\Psi_{\mathrm{QPIR}}^{(\mathrm{m})}\right) & :=\mathbb{E}_{\pi \in \mathrm{S}_{\mathrm{n}}} I\left(K ; Q_{\pi, \mathrm{t}}\right),
\end{aligned}
$$

and QPIR capacities $\tilde{C}_{\text {exact,t }}^{\alpha, \beta, \gamma, \theta}$ and $\tilde{C}_{\text {asymp,t }}^{\alpha, \beta, \gamma, \theta}$ are defined the same as (18) and (19) except that the security measures $P_{\text {err }}\left(\Psi_{\mathrm{QPIR}}^{(\mathrm{m})}\right), S_{\text {serv }}\left(\Psi_{\mathrm{QPIR}}^{(\mathrm{m})}\right), S_{\text {user }}^{(\mathrm{t})}\left(\Psi_{\mathrm{QPIR}}^{(\mathrm{m})}\right)$ are replaced by $\tilde{P}_{\text {err }}\left(\Psi_{\mathrm{QPIR}}^{(\mathrm{m})}\right), \tilde{S}_{\mathrm{serv}}\left(\Psi_{\mathrm{QPIR}}^{(\mathrm{m})}\right), \tilde{S}_{\mathrm{user}}^{(\mathrm{t})}\left(\Psi_{\mathrm{QPIR}}^{(\mathrm{m})}\right)$. Then, similar to Theorem 11, the average capacity is derived as

$$
\begin{array}{ll}
\tilde{C}_{\text {asymp }, \mathrm{t}}^{\alpha, \beta, \gamma, \theta}=\tilde{C}_{\text {exact }, \mathrm{t}}^{\alpha, \beta, \gamma, \theta}=1 & \text { if } 1 \leq \mathrm{t} \leq \frac{\mathrm{n}}{2}, \\
\tilde{C}_{\text {asymp }, \mathrm{t}}^{0, \beta, 0, \theta}=\tilde{C}_{\text {exact }, \mathrm{t}}^{\alpha, 0,0, \theta}=\frac{2(\mathrm{n}-\mathrm{t})}{\mathrm{n}} & \text { if } \frac{\mathrm{n}}{2}<\mathrm{t}<\mathrm{n} .
\end{array}
$$

For the achievability proof of (80) and (81), the QPIR protocol in Section $\nabla$ achieves the capacity.

The converse bounds are also proved similar to the case of the worst-case security. The converse bounds are written for any $\alpha \in[0,1)$ and any $\beta, \gamma, \theta \in[0, \infty)$ as

$$
\begin{aligned}
\tilde{C}_{\text {asymp,t }}^{\alpha, \beta, \gamma, \theta} \leq 1 & \text { if } 1 \leq \mathrm{t} \leq \frac{\mathrm{n}}{2}, \\
\tilde{C}_{\text {exact }, \mathrm{t}}^{\alpha, 0,0, \theta} \leq \frac{2(\mathrm{n}-\mathrm{t})}{\mathrm{n}} & \text { if } \frac{\mathrm{n}}{2}<\mathrm{t}<\mathrm{n}, \\
\tilde{C}_{\text {asymp } \mathrm{t}}^{0, \beta, 0, \theta} \leq \frac{2(\mathrm{n}-\mathrm{t})}{\mathrm{n}} & \text { if } \frac{\mathrm{n}}{2}<\mathrm{t}<\mathrm{n} .
\end{aligned}
$$

First, the converse bound (84) is proved by the same steps as Section $\nabla I$ except for the following part. In Section DI] Eq. (73) is written as

$$
\begin{aligned}
& \left(1-P_{\mathrm{err}, k, q}\left(\Psi_{\mathrm{QPIR}}^{(\mathrm{m})}\right)\right) \log \mathrm{m} \\
& \leq D\left(\tilde{\rho}\left(M_{k} \mid q\right) \| \tilde{\rho}\left(q_{\mathrm{t}}\right)\right)+h_{2}\left(P_{\mathrm{err}, k, q}\left(\Psi_{\mathrm{QPIR}}^{(\mathrm{m})}\right)\right),
\end{aligned}
$$

and by taking the expectation of (73) with respect to $Q$, we obtain (74). Similarly, we take the expectation of (73) with respect to $K, Q$ and then, we obtain

$$
\begin{aligned}
& \left(1-\tilde{P}_{\mathrm{err}}\left(\Psi_{\mathrm{QPIR}}^{(\mathrm{m})}\right)\right) \log \mathrm{m} \\
& \leq \mathbb{E}_{K, Q} D\left(\tilde{\rho}\left(M_{K} \mid Q\right) \| \tilde{\rho}\left(Q_{\mathrm{t}}\right) \mid K\right)+h_{2}\left(\tilde{P}_{\mathrm{err}}\left(\Psi_{\mathrm{QPIR}}^{(\mathrm{m})}\right)\right) .
\end{aligned}
$$

Then, by the similar steps as Section VI, the converse bound (84) is proved.

For the converse bounds (82) and (83), in the last steps of the proofs in Appendix $\mathrm{F}$ we replace (116) and (123) by

$$
1-\tilde{P}_{\mathrm{err}}\left(\Psi_{\mathrm{QPIR}}^{\left(\mathrm{m}_{\ell}\right)}\right) \leq 1-\inf _{k, q} P_{\mathrm{err}, k, q}\left(\Psi_{\mathrm{QPIR}}^{\left(\mathrm{m}_{\ell}\right)}\right) .
$$

Then, the converse bounds (82) and (83) are proved in the same way.

\section{APPENDIX B \\ ProOf OF PROPOSITION 1}

We concretely construct the subgroup $S(\mathrm{~V})$ as follows. From (25), all elements of $S(\mathrm{~V})$ are commutative regardless of the choice of $c_{\mathbf{v}}$. Since $I \in S(\mathrm{~V})$, we set $\mathbf{W}(\mathbf{0})=I$, i.e., $c_{0}=1$. Then, it is enough to choose $c_{\mathrm{v}}$ so that $S(\mathrm{~V})$ satisfies the closure for the multiplication.

We choose $\left\{c_{\mathbf{v}} \in \mathbb{C} \mid \mathbf{v} \in \mathrm{V}\right\}$ as follows. For a fixed basis $\mathbf{v}_{1}, \ldots, \mathbf{v}_{d}$ of $\mathrm{V}$, we choose $c_{\mathbf{v}_{i}}$ as follows: if $p>2$, choose $c_{\mathbf{v}_{i}}$ as a $p$-th root of unity, i.e., $c_{\mathbf{v}_{i}}=\omega^{k}$ for some integer $k$; if $p=2$, choose $c_{\mathbf{v}_{i}}$ as

$$
c_{\mathbf{v}_{i}}= \pm \sqrt{-1}^{\left\langle\mathbf{b}_{i}, \mathbf{a}_{i}\right\rangle}= \begin{cases} \pm 1 & \text { if }\left\langle\mathbf{b}_{i}, \mathbf{a}_{i}\right\rangle=0, \\ \pm \sqrt{-1} & \text { if }\left\langle\mathbf{b}_{i}, \mathbf{a}_{i}\right\rangle=1,\end{cases}
$$

where $\mathbf{a}_{i}, \mathbf{b}_{i}$ are vectors in $\mathbb{F}_{q}^{n}$ such that $\left(\mathbf{a}_{i}, \mathbf{b}_{i}\right)=\mathbf{v}_{i}$. For any $\mathbf{v}=\sum_{i} a_{i} \mathbf{v}_{i} \in \mathrm{V}$, we choose $c_{\mathbf{v}}$ by the relation

$$
\mathbf{W}(\mathbf{v})=\mathbf{W}\left(\mathbf{v}_{1}\right)^{a_{1}} \cdots \mathbf{W}\left(\mathbf{v}_{d}\right)^{a_{d}} .
$$

Next, we prove the closure for the multiplication in $S(\mathrm{~V})$ by the above choice of $c_{\mathrm{v}}$. For any basis element $\mathbf{v}_{i}$, we have

$$
\begin{aligned}
\mathbf{W}\left(\mathbf{v}_{i}\right)^{p} & =c_{\mathbf{v}_{i}}^{p} \tilde{\mathbf{W}}\left(\mathbf{v}_{i}\right)^{p} \stackrel{(a)}{=} c_{\mathbf{v}_{i}}^{p} \omega^{p(p-1)\left\langle\mathbf{b}_{i}, \mathbf{a}_{i}\right\rangle / 2} \tilde{\mathbf{W}}\left(p \mathbf{v}_{i}\right) \\
& =c_{\mathbf{v}_{i}}^{p} \omega^{p(p-1)\left\langle\mathbf{b}_{i}, \mathbf{a}_{i}\right\rangle / 2} I=I,
\end{aligned}
$$

where $(a)$ follows from (26). Then, we can confirm the closure for the multiplication as

$$
\begin{aligned}
& \mathbf{W}(\mathbf{v}) \mathbf{W}\left(\mathbf{v}^{\prime}\right) \\
& =\mathbf{W}\left(\mathbf{v}_{1}\right)^{a_{1}} \cdots \mathbf{W}\left(\mathbf{v}_{d}\right)^{a_{d}} \mathbf{W}\left(\mathbf{v}_{1}\right)^{a_{1}^{\prime}} \cdots \mathbf{W}\left(\mathbf{v}_{d}\right)^{a_{d}^{\prime}} \\
& =\mathbf{W}\left(\mathbf{v}_{1}\right)^{a_{1}+a_{1}^{\prime}} \cdots \mathbf{W}\left(\mathbf{v}_{d}\right)^{a_{d}+a_{d}^{\prime}} \\
& =\mathbf{W}\left(\mathbf{v}_{1}\right)^{a_{1}+a_{1}^{\prime} \bmod p} \cdots \mathbf{W}\left(\mathbf{v}_{d}\right)^{a_{d}+a_{d}^{\prime} \bmod p} \\
& =\mathbf{W}\left(\mathbf{v}+\mathbf{v}^{\prime}\right)
\end{aligned}
$$

for any $\mathbf{v}, \mathbf{v}^{\prime} \in \mathrm{V}$, where the equality (88) is from the commutative property of $S(\mathrm{~V})$ and the equality 897 is from (87). Thus, $S(\mathrm{~V})$ is a commutative subgroup of $\mathrm{HW}_{q}^{n}$ not containing $c I$ for any $c \neq 0$, i.e., a stabilizer.

Alternatively, if $p>2$, the set $S(\mathrm{~V})$ is a stabilizer by choosing $c_{(\mathbf{a}, \mathbf{b})}=\left(\omega^{(p+1) / 2}\right)^{\langle\mathbf{a}, \mathbf{b}\rangle}$ since

$\mathbf{W}(\mathbf{a}, \mathbf{b}) \mathbf{W}(\mathbf{c}, \mathbf{d})=\left(\omega^{(p+1) / 2}\right)^{\langle(\mathbf{a}, \mathbf{b}), J(\mathbf{c}, \mathbf{d})\rangle} \mathbf{W}(\mathbf{a}+\mathbf{c}, \mathbf{b}+\mathbf{d})$

for any $(\mathbf{a}, \mathbf{b}),(\mathbf{c}, \mathbf{d}) \in \mathbb{F}_{q}^{n}$ and $\mathrm{V}$ is self-orthogonal.

\section{APPENDIX C \\ PROOF OF PROPOSITION 2}

Let $\mathrm{V}$ be a self-orthogonal $d$-dimensional subspace of $\mathbb{F}_{q}^{2 n}$ and $S(\mathrm{~V})$ be a stabilizer defined by (27). Notice the following facts.

Fact 1) $\mathbf{W}(\mathbf{v}) \mathbf{W}\left(\mathbf{v}^{\prime}\right)=\mathbf{W}\left(\mathbf{v}+\mathbf{v}^{\prime}\right)$ for any $\mathbf{v}, \mathbf{v}^{\prime} \in \mathrm{V}$ by the closure for the multiplication of $S(\mathrm{~V})$,

Fact 2) All eigenvalues of $\mathbf{W}(\mathbf{v})$ are in $\left\{\omega^{k} \mid k \in \mathbb{F}_{p}\right\}$, since $(\mathbf{W}(\mathbf{v}))^{p}=\mathbf{W}(p \mathbf{v})=\mathbf{W}(\mathbf{0})=I_{q^{n}}$ for any $\mathbf{v} \in \mathrm{V}$.

Fact 3) All elements of $S(\mathrm{~V})$ are simultaneously diagonalized, since $S(\mathrm{~V})$ is a commutative group. 
First, we prove 1) of the proposition. By Facts 2 and 3, we have the simultaneous decomposition of all elements $\mathbf{W}(\mathbf{v}) \in$ $S(\mathrm{~V})$ as

$$
\mathbf{W}(\mathbf{v})=\sum_{f: \mathrm{V} \rightarrow \mathbb{F}_{p}} \omega^{f(\mathbf{v})} P_{f}^{\mathrm{V}} \quad(\forall \mathbf{v} \in \mathrm{V}),
$$

where the summation is taken for all maps $f$ from $\mathrm{V}$ to $\mathbb{F}_{p}$ and $\left\{P_{f}^{\mathrm{V}}\right\}$ are orthogonal projections (including the zero matrix) such that

$$
\begin{aligned}
P_{f}^{\mathrm{V}} P_{f^{\prime}}^{\mathrm{V}} & =0 \text { for any } f \neq f^{\prime}, \\
\sum_{f \in \mathrm{V}^{*}} & =I_{\mathcal{H}^{\otimes n}} .
\end{aligned}
$$

Let $\mathrm{V}^{*}$ be the space of linear maps from $\mathrm{V}$ to $\mathbb{F}_{p}$. Since Fact 1 implies $\omega^{f(\mathbf{v})+f\left(\mathbf{v}^{\prime}\right)} P_{f}^{\mathrm{V}}=\omega^{f\left(\mathbf{v}+\mathbf{v}^{\prime}\right)} P_{f}^{\mathrm{V}}$ for any $\mathbf{v} \in \mathrm{V}$ and $f: \mathrm{V} \rightarrow \mathbb{F}_{p}$, we have $P_{f}^{\mathrm{V}}=0$ for any $f \notin \mathrm{V}^{*}$. Thus, (90) is written as

$$
\mathbf{W}(\mathbf{v})=\sum_{f \in \mathrm{V}^{*}} \omega^{f(\mathbf{v})} P_{f}^{\mathrm{V}} \quad(\forall \mathbf{v} \in \mathrm{V}) .
$$

Furthermore, the space $\mathrm{V}^{*}$ is isomorphic to $\mathbb{F}_{q}^{2 n} / \mathrm{V}^{\perp_{J}}$ by the following identification: we identify $f \in \mathrm{V}^{*}$ and $[\mathbf{w}]:=$ $\mathbf{w}+\mathrm{V}^{\perp_{J}} \in \mathbb{F}_{q}^{2 n} / \mathrm{V}^{\perp_{J}}$ if $f(\mathbf{v})=\langle\mathbf{v}, J \mathbf{w}\rangle$ for any $\mathbf{v} \in \mathrm{V}$. Therefore, we denote $P_{[\mathbf{w}]}^{\mathrm{V}}:=P_{f}^{\mathrm{V}}$ if $f$ and $[\mathbf{w}]$ are identical and Eq. (93) is written as

$$
\mathbf{W}(\mathbf{v})=\sum_{[\mathbf{w}] \in \mathbb{F}_{q}^{2 n} / \mathrm{V}^{\perp J}} \omega^{\langle\mathbf{v}, J \mathbf{w}\rangle} P_{[\mathbf{w}]}^{\mathrm{V}} \quad(\forall \mathbf{v} \in \mathrm{V}),
$$

which implies 1) of the proposition. The uniqueness of the decomposition (94) is from the uniqueness of the eigendecomposition.

Next, we prove 2) of the proposition. Let $\mathcal{H}_{[\mathbf{w}]}^{\mathrm{V}}:=\operatorname{Im} P_{[\mathbf{w}]}^{\mathrm{V}}$. For any $\mathbf{v} \in \mathrm{V}$, we have

$$
\begin{aligned}
\mathbf{W}(\mathbf{v}) \mathbf{W}(\mathbf{w}) \mathcal{H}_{\left[\mathbf{w}^{\prime}\right]}^{\mathrm{V}} & \stackrel{(a)}{=} \omega^{\langle\mathbf{v}, J \mathbf{w}\rangle} \mathbf{W}(\mathbf{w}) \mathbf{W}(\mathbf{v}) \mathcal{H}_{\left[\mathbf{w}^{\prime}\right]}^{\mathrm{V}} \\
& \stackrel{(b)}{=} \omega^{\left\langle\mathbf{v}, J\left(\mathbf{w}+\mathbf{w}^{\prime}\right)\right\rangle} \mathbf{W}(\mathbf{w}) \mathcal{H}_{\left[\mathbf{w}^{\prime}\right]}^{\mathrm{V}},
\end{aligned}
$$

where $(a)$ is from

$$
\mathbf{W}(\mathbf{v}) \mathbf{W}(\mathbf{w})=\omega^{\langle\mathbf{v}, J \mathbf{w}\rangle} \mathbf{W}(\mathbf{w}) \mathbf{W}(\mathbf{v}),
$$

which follows from (25), and $(b)$ is from

$$
\mathbf{W}(\mathbf{v}) \mathcal{H}_{\left[\mathbf{w}^{\prime}\right]}^{\mathrm{V}}=\omega^{\left\langle\mathbf{v}, J \mathbf{w}^{\prime}\right\rangle} \mathcal{H}_{\left[\mathbf{w}^{\prime}\right]}^{\mathrm{V}},
$$

which follows from 94). Since 96 implies that $\mathbf{W}(\mathbf{v})$ maps $\mathbf{W}(\mathbf{w}) \mathcal{H}_{\left[\mathbf{w}^{\prime}\right]}^{\mathrm{V}}$ to $\omega^{\left\langle\mathbf{v}, J\left(\mathbf{w}+\mathbf{w}^{\prime}\right)\right\rangle} \mathbf{W}(\mathbf{w}) \mathcal{H}_{\left[\mathbf{w}^{\prime}\right]}^{\mathrm{V}}$, we have $\mathbf{W}(\mathbf{w}) \mathcal{H}_{\left[\mathbf{w}^{\prime}\right]}^{\mathrm{V}} \subseteq \mathcal{H}_{\left[\mathbf{w}+\mathbf{w}^{\prime}\right]}^{\mathrm{V}}$ from (94). Conversely, we also have $\mathbf{W}(-\mathbf{w}) \mathcal{H}_{\left[\mathbf{w}+\mathbf{w}^{\prime}\right]}^{\mathrm{V}} \subseteq \mathcal{H}_{\left[\mathbf{w}^{\prime}\right]}^{\mathrm{V}}$. Thus, we have $\operatorname{dim} \mathcal{H}_{\left[\mathbf{w}^{\prime}\right]}^{\mathrm{V}}=$ $\operatorname{dim} \mathcal{H}_{\left[\mathbf{w}+\mathbf{w}^{\prime}\right]}^{\mathrm{V}}$ and therefore, obtain the desired relation $\mathbf{W}(\mathbf{w}) \mathcal{H}_{\left[\mathbf{w}^{\prime}\right]}^{\mathrm{V}}=\mathcal{H}_{\left[\mathbf{w}+\mathbf{w}^{\prime}\right]}^{\mathrm{V}}$.

Lastly, we prove 3) of the proposition. By 2) of the proposition, all spaces $\mathcal{H}_{[\mathbf{w}]}^{\mathrm{V}}$ have the same dimension. Therefore, we have

$$
\operatorname{dim} \mathcal{H}_{[\mathbf{w}]}^{\mathrm{V}}=\frac{\operatorname{dim} \mathcal{H}^{\otimes n}}{\left|\mathbb{F}_{q}^{2 n} / \mathrm{V}^{\perp J}\right|}=\frac{\operatorname{dim} \mathcal{H}^{\otimes n}}{|\mathrm{~V}|}=q^{n-d} .
$$

\section{APPENDIX D \\ PROOF OF LEMMA 2}

For the proof of Lemma 2, we prepare the following proposition.

Proposition 4. Let $\mathbb{F}_{q^{\prime}}$ be the finite field of order $q^{\prime}$ and $\mathbb{F}_{q}$ be an extension field of $\mathbb{F}_{q^{\prime}}$ such that $\mathbb{F}_{q^{\prime}}\left(\alpha_{1}, \ldots, \alpha_{k-2}\right)$, where $\alpha_{i} \notin \mathbb{F}_{q^{\prime}}\left(\alpha_{1}, \ldots, \alpha_{i-1}\right)$ for any $i$. Given two positive integers $r<k$, we define a matrix $A=\left(a_{i j}\right) \in \mathbb{F}_{q}^{(k-r) \times r}$ such that

$$
\begin{aligned}
& a_{11}=1, \\
& a_{i j} \in \mathbb{F}_{q^{\prime}}\left(\alpha_{1}, \ldots, \alpha_{i+j-2}\right) \backslash \mathbb{F}_{q^{\prime}}\left(\alpha_{1}, \ldots, \alpha_{i+j-3}\right)
\end{aligned}
$$

if $(i, j) \neq(1,1)$. Then, any $r$ row vectors of

$$
\bar{A}:=\left(\begin{array}{c}
A \\
I_{r}
\end{array}\right) \in \mathbb{F}_{q}^{k \times r}
$$

are linearly independent. In particular, when $k=2 r$, the square matrix $A \in \mathbb{F}_{q}^{r \times r}$ is invertible.

Proof of Proposition 4. Before we give the proof, we introduce the following notation: For an $n \times m$ matrix $M=\left(m_{i j}\right), S \subset\{1, \ldots, n\}$ and $T \subset\{1, \ldots, m\}$, define a submatrix $M(S, T):=\left(m_{i j}\right)_{i \in S, j \in T \text {. }}$.

Let $S \subset\{1, \ldots, k-r\}$ and $T \subset\{1, \ldots, r\}$ be subsets such that $|S|+|T|=r$. Choose $r$ row vectors of $\bar{A}$ as

$$
\bar{A}(S \cup(k-r+T),\{1, \ldots, r\})=\left(\begin{array}{c}
A(S,\{1, \ldots, r\}) \\
I_{r}(T,\{1, \ldots, r\})
\end{array}\right) .
$$

The row vectors of $\bar{A}(S \cup(k-r+T),\{1, \ldots, r\})$ are linearly independent if and only if $A\left(S, T^{c}\right) \in \mathbb{F}_{q}^{|S| \times|S|}$ is invertible, where $T^{c}:=\{1, \ldots, r\} \backslash T$. Therefore, we show in the following that the determinant of $A\left(S, T^{c}\right)$ is nonzero.

From the definition of $A$ in (98), the $\quad(|S|,|S|) \quad$ element $\quad a_{\max S, \max T^{c}} \quad$ of $A\left(S, T^{c}\right) \quad$ is in $\quad \mathbb{F}_{q^{\prime}}\left(\alpha_{1}, \ldots, \alpha_{\max S+\max T^{c}-2}\right) \quad$ । $\mathbb{F}_{q^{\prime}}\left(\alpha_{1}, \ldots, \alpha_{\max S+\max T^{c}-3}\right)$ but the other $|S|^{2}-1$ elements are in $\mathbb{F}_{q^{\prime}}\left(\alpha_{1}, \ldots, \alpha_{\max S+\max T^{c}-3}\right)$. Thus, by the cofactor expansion of the determinant, i.e., $\operatorname{det} M=\sum_{j}(-1)^{i+j} m_{i, j} M_{i, j}$ for a matrix $M=\left(m_{i j}\right)$ and its $i, j$ minor $M_{i, j}$, we have

$$
\begin{aligned}
& \operatorname{det} A\left(S, T^{c}\right) \\
& =a_{\max S, \max T^{c}} \cdot \operatorname{det} A\left(S, T^{c}\right)_{|S|,|S|}+x \\
& =a_{\max S, \max T^{c}} \cdot \operatorname{det} A\left(S \backslash\{\max S\}, T^{c} \backslash\left\{\max T^{c}\right\}\right)+x
\end{aligned}
$$

with some $x \in \mathbb{F}_{q^{\prime}}\left(\alpha_{1}, \ldots, \alpha_{\max S+\max T^{c}-3}\right)$. If

$$
\operatorname{det} A\left(S \backslash\{\max S\}, T^{c} \backslash\left\{\max T^{c}\right\}\right) \neq 0,
$$

then $\operatorname{det} A\left(S, T^{c}\right) \neq 0$. Thus, by induction, we have $\operatorname{det} A\left(S, T^{c}\right) \neq 0$ since $\operatorname{det} A\left(\{\min S\},\left\{\min T^{c}\right\}\right)=$ $a_{\min S, \min T^{c}} \neq 0$.

Remark 9. Proposition 4 is a slight generalization of the construction [43, Appendix A], which proposed the same construction only for $a_{i j}=\alpha_{i+j-2}$ in (98).

Now, we prove Lemma 2 Let $S \in \mathbb{F}_{q}^{2 n \times 2 n}$ be a symplectic matrix, i.e., $S^{\top} J S=J$, and $\mathbf{s}_{i} \in \mathbb{F}_{q}^{2 n}$ be the $i$-th column 
vector of $S$. Then, the following $\mathbf{v}_{1}, \ldots, \mathbf{v}_{2 t} \in \mathbb{F}_{q}^{2 n}$ satisfy condition (b):

$$
\begin{aligned}
\left(\mathbf{v}_{1}, \ldots, \mathbf{v}_{2 n-2 t}\right) & :=\left(\mathbf{s}_{2 t-n+1}, \ldots, \mathbf{s}_{n}\right) \\
\left(\mathbf{v}_{2 n-2 t+1}, \ldots, \mathbf{v}_{2 t}\right) & :=\left(\mathbf{s}_{1}, \ldots, \mathbf{s}_{2 t-n}, \mathbf{s}_{n+1}, \ldots, \mathbf{s}_{2 t}\right) .
\end{aligned}
$$

Therefore, in the following, we prove that there exists a symplectic matrix $S=\left(\mathbf{s}_{1}, \ldots, \mathbf{s}_{2 n}\right)$ such that the row vectors of $S^{\prime}:=\left(\mathbf{s}_{1}, \ldots, \mathbf{s}_{2 t}\right)$ satisfy condition $(\mathrm{a})$.

First, we construct a symplectic matrix as follows. For convenience, let $\alpha_{0}:=1$. Define two square symmetric matrices $A=\left(a_{i j}\right), B=\left(b_{i j}\right) \in \mathbb{F}_{q}^{n \times n}$ as

$$
a_{i j}=\alpha_{i+j-2}, \quad b_{i j}=\alpha_{i+j-2+(2 t-n)},
$$

i.e.,

$$
\begin{aligned}
A & =\left(\begin{array}{cccc}
\alpha_{0} & \alpha_{1} & \cdots & \alpha_{n-1} \\
\alpha_{1} & \alpha_{2} & \cdots & \alpha_{n-2} \\
\vdots & \vdots & \ddots & \vdots \\
\alpha_{n-1} & \alpha_{n} & \cdots & \alpha_{2 n-2}
\end{array}\right), \\
B & =\left(\begin{array}{cccc}
\alpha_{2 t-n} & \alpha_{2 t-n+1} & \cdots & \alpha_{2 t-1} \\
\alpha_{2 t-n+1} & \alpha_{2 t-n+2} & \cdots & \alpha_{2 t} \\
\vdots & \vdots & \ddots & \vdots \\
\alpha_{2 t-1} & \alpha_{2 t} & \cdots & \alpha_{n+2 t-2}
\end{array}\right) .
\end{aligned}
$$

Since the matrices

$$
\left(\begin{array}{cc}
I_{n} & X \\
0 & I_{n}
\end{array}\right),\left(\begin{array}{cc}
I_{n} & 0 \\
X & I_{n}
\end{array}\right)
$$

are symplectic matrices for any symmetric matrix $X$, and the multiple of two symplectic matrices is a symplectic matrix [37, Section 8.2.2], the matrix

$$
S=\left(\begin{array}{cc}
I_{n}+B A^{-1} & B \\
A^{-1} & I_{n}
\end{array}\right)=\left(\begin{array}{cc}
I_{n} & B \\
0 & I_{n}
\end{array}\right)\left(\begin{array}{cc}
I_{n} & 0 \\
A^{-1} & I_{n}
\end{array}\right)
$$

is a symplectic matrix, where the inverse $A^{-1}$ exists from Proposition 4. With the notation $B=\left(B_{1}, B_{2}\right) \in \mathbb{F}_{q}^{n \times(2 t-n)} \times$ $\mathbb{F}_{q}^{n \times(2 n-2 t)}$, we have

$$
S^{\prime}:=\left(\mathbf{s}_{1}, \ldots, \mathbf{s}_{2 t}\right)=\left(\begin{array}{c|c}
I_{n}+B A^{-1} & B_{1} \\
\hline A^{-1} & \begin{array}{c}
I_{2 t-n} \\
0
\end{array}
\end{array}\right) .
$$

Now, we prove that the row vectors of $S^{\prime}$ satisfy condition (a). Since (i) the right multiplication of invertible matrices and (ii) elementary column operations do not change the linear independence of the row vectors, we manipulate the matrix $S^{\prime}$ in the following way:

$$
\begin{aligned}
& S^{\prime}=\left(\begin{array}{c|c}
I_{n}+B A^{-1} & B_{1} \\
\hline A^{-1} & \begin{array}{c}
I_{2 t-n} \\
0
\end{array}
\end{array}\right) \\
& \stackrel{\text { (i) }}{\longrightarrow}\left(\begin{array}{c|c}
I_{n}+B A^{-1} & B_{1} \\
\hline A^{-1} & \begin{array}{c}
I_{2 t-n} \\
0
\end{array}
\end{array}\right)\left(\begin{array}{cc}
A & 0 \\
0 & I_{2 t-n}
\end{array}\right)
\end{aligned}
$$

$$
\begin{aligned}
& =\left(\begin{array}{c|c}
A+B & B_{1} \\
\hline I_{n} & \begin{array}{c}
I_{2 t-n} \\
0
\end{array}
\end{array}\right) \\
& =\left(\begin{array}{cc|c}
A_{1}+B_{1} & A_{2}+B_{2} & B_{1} \\
\hline I_{2 t-n} & 0 & I_{2 t-n} \\
0 & I_{2 n-2 t} & 0
\end{array}\right) \\
& \stackrel{\text { (ii) }}{\longrightarrow}\left(\begin{array}{cc|c}
A_{1} & A_{2}+B_{2} & B_{1} \\
\hline 0 & 0 & I_{2 t-n} \\
0 & I_{2 n-2 t} & 0
\end{array}\right) \\
& \stackrel{\text { (ii) }}{\longrightarrow}\left(\begin{array}{ccc}
A_{1} & B_{1} & A_{2}+B_{2} \\
\hline 0 & I_{2 t-n} & 0 \\
0 & 0 & I_{2 n-2 t}
\end{array}\right)=: S^{\prime \prime} \text {, }
\end{aligned}
$$

where $A=\left(A_{1}, A_{2}\right) \in \mathbb{F}_{q}^{n \times(2 t-n)} \times \mathbb{F}_{q}^{n \times(2 n-2 t)}$. By the above transformation, the linear independence of the row vectors of $S^{\prime}$ is equivalent to that of $S^{\prime \prime}$. Let

$$
S^{\prime \prime \prime}:=\left(\begin{array}{ccc}
A_{1} & B_{1} & A_{2}+B_{2} \\
\hline I_{2 t-n} & 0 & 0 \\
0 & I_{2 t-n} & 0 \\
0 & 0 & I_{2 n-2 t}
\end{array}\right)
$$

by adding the row vectors $\left(I_{2 t-n}, 0,0\right)$ to $S^{\prime \prime}$. If any $2 t$ row vectors of $S^{\prime \prime \prime}$ are linearly independent, then $S^{\prime \prime}$ and $S^{\prime}$ also satisfy the same property. Note that we can apply Proposition 4 to $S^{\prime \prime \prime}$ since the matrices $A_{1}, B_{1}, A_{2}+B_{2}$ are written as

$$
\begin{aligned}
A_{1} & =\left(\begin{array}{ccc}
\alpha_{0} & \cdots & \alpha_{2 t-n-1} \\
\vdots & \ddots & \vdots \\
\alpha_{n-1} & \cdots & \alpha_{2 t-2}
\end{array}\right) \\
B_{1} & =\left(\begin{array}{ccc}
\alpha_{2 t-n} & \cdots & \alpha_{4 t-2 n-1} \\
\vdots & \ddots & \vdots \\
\alpha_{2 t-1} & \cdots & \alpha_{4 t-n-2}
\end{array}\right) \\
A_{2}+B_{2} & =\left(\begin{array}{ccc}
\alpha_{4 t-2 n}+\alpha_{2 t-n} & \cdots & \alpha_{2 t-1}+\alpha_{n-1} \\
\vdots & \ddots & \vdots \\
\alpha_{4 t-n-1}+\alpha_{2 t-1} & \cdots & \alpha_{n+2 t-2}+\alpha_{2 n-2}
\end{array}\right)
\end{aligned}
$$

and therefore $\left(A_{1}, B_{1}, A_{2}+B_{2}\right)$ satisfies the condition (98). The application of Proposition 4 to $S^{\prime \prime \prime}$ shows that any $2 t$ row vectors of $S^{\prime \prime \prime}$ are linearly independent. Thus, the matrix $S^{\prime}$ also satisfies the same property as $S^{\prime \prime \prime}$, which implies condition (a). This finishes the proof of the desired statement.

\section{APPENDIX E}

\section{PROOF OF LEMMA 4}

Throughout this section, we use the following notation. For random variables $X$ and $Y$, we denote by $p_{X}$ the probability distribution of $X$, by $p_{X \mid Y}$ the distribution of $X$ conditioned by $Y$, by $p_{X \mid Y=y}$ the distribution of $X$ conditioned by $Y=y$. 
We also denote $p_{X=x}=\operatorname{Pr}[X=x]$ and $p_{X=x \mid Y=y}=\operatorname{Pr}[X=$ $x \mid Y=y]$ for simplicity.

For the proof of Lemma4, we prepare the following lemma.

Lemma 5. The server secrecy $S_{\text {serv }}\left(\Psi_{\mathrm{QPIR}}^{(\mathrm{m})}\right) \leq \beta$ implies

$$
I\left(M_{k}^{c} ; \mathcal{A} Q \mid K=k\right)_{\rho(M, Q)} \leq \beta .
$$

The user t-secrecy $S_{\mathrm{user}}^{(\mathrm{t})}\left(\Psi_{\mathrm{QPIR}}^{(\mathrm{m})}\right) \leq \gamma$ implies

$$
\max _{i \neq k \in\{1, \ldots, f\}, \pi \in \mathrm{S}_{\mathrm{n}}} d\left(p_{Q_{\mathrm{t}} \mid K=k}, p_{Q_{\mathrm{t}} \mid K=i}\right) \leq \sqrt{2 \mathrm{f} \gamma},
$$

where $d(\cdot, \cdot)$ is the variational distance $d(p, q):=(1 / 2)$. $\sum_{j}\left|p_{j}-q_{j}\right|$ for probability distributions $p, q$.

Proof: The relation (102) is proved as follows:

$$
\begin{aligned}
& I\left(M_{k}^{c} ; \mathcal{A} Q \mid K=k\right)_{\rho(M, Q)} \\
& =I\left(M_{k}^{c} ; \mathcal{A} \mid Q, K=k\right)_{\rho(M, Q)}+I\left(M_{k}^{c} ; Q \mid K=k\right)_{\rho(M, Q)} \\
& =I\left(M_{k}^{c} ; \mathcal{A} \mid Q, K=k\right)_{\rho(M, Q)} \\
& =\sum_{q} p_{Q=q \mid K=k} \cdot I\left(M_{k}^{c} ; \mathcal{A} \mid Q=q, K=k\right)_{\rho(M, Q)} \leq \beta,
\end{aligned}
$$

where the equality (104) holds because $Q$ is independent of $M_{k}^{c}$.

The relation (103) is proved as follows. For any $\pi \in \mathrm{S}_{\mathrm{n}}$ and any $k \in\{1, \ldots, \mathrm{f}\}$,

$$
\begin{aligned}
\gamma & \geq I\left(K ; Q_{\mathrm{t}}\right)=D\left(p_{K Q_{\mathrm{t}}} \| p_{K} \times p_{Q_{\mathrm{t}}}\right) \\
& =\frac{1}{\mathrm{f}} \sum_{k^{\prime}} D\left(p_{Q_{\mathrm{t}} \mid K=k^{\prime}} \| p_{Q_{\mathrm{t}}}\right) \stackrel{(a)}{\geq} \frac{2}{\mathrm{f}} \sum_{k^{\prime}} d^{2}\left(p_{Q_{\mathrm{t}} \mid K=k^{\prime}}, p_{Q}\right) \\
& \geq \frac{2}{\mathrm{f}} d^{2}\left(p_{Q_{\mathrm{t}} \mid K=k}, p_{Q_{\mathrm{t}}}\right),
\end{aligned}
$$

where the inequality $(a)$ follows from Pinsker inequality. Thus, for any $i, k \in\{1, \ldots, \mathrm{f}\}$, we have

$$
\begin{aligned}
\sqrt{2 \mathrm{f} \gamma} & \geq d\left(p_{Q_{\mathrm{t}} \mid K=k}, p_{Q_{\mathrm{t}}}\right)+d\left(p_{Q_{\mathrm{t}}}, p_{Q_{\mathrm{t}} \mid K=i}\right) \\
& \geq d\left(p_{Q_{\mathrm{t}} \mid K=k}, p_{Q_{\mathrm{t}} \mid K=i}\right),
\end{aligned}
$$

which implies (103).

Now, we prove Lemma 4 Let $k \neq i \in\{1, \ldots, \mathrm{f}\}$. Then, we obtain Lemma 4 as

$$
\begin{aligned}
& I\left(M_{k} ; \mathcal{A}_{\mathrm{t}} \mid Q_{\mathrm{t}}, K=k\right)_{\rho\left(M, Q_{\mathrm{t}}\right)} \\
& \leq I\left(M_{k} ; \mathcal{A}_{\mathrm{t}} Q_{\mathrm{t}} \mid K=k\right)_{\rho\left(M, Q_{\mathrm{t}}\right)} \\
& \stackrel{(a)}{\leq} I\left(M_{k} ; \mathcal{A}_{\mathrm{t}} Q_{\mathrm{t}} \mid K=i\right)_{\rho\left(M, Q_{\mathrm{t}}\right)}+g(\mathrm{~m}, \gamma) \\
& \leq I\left(M_{i}^{c} ; \mathcal{A} Q \mid K=i\right)_{\rho(M, Q)}+g(\mathrm{~m}, \gamma) \\
& \stackrel{(b)}{\leq} \beta+g(\mathrm{~m}, \gamma),
\end{aligned}
$$

where the inequality $(b)$ follows from 102 ) of Lemma 5 .

The inequality $(a)$ is derived as follows. When we define $\tilde{\rho}\left(M, Q_{\mathrm{t}} \mid k\right):=\sum_{m, q_{\mathrm{t}}}\left(1 / \mathrm{m}^{\mathrm{f}}\right) \cdot p_{Q_{\mathrm{t}}=q_{\mathrm{t}} \mid K=k} \cdot\left|m, q_{\mathrm{t}}\right\rangle\left\langle m, q_{\mathrm{t}}\right| \otimes$ $\rho\left(m, q_{\mathrm{t}}\right)$ for $k \in\{1, \ldots, \mathrm{f}\}$, the inequality (103) of Lemma 5 implies that

$$
d\left(\tilde{\rho}\left(M, Q_{\mathrm{t}} \mid k\right), \tilde{\rho}\left(M, Q_{\mathbf{t}} \mid i\right)\right) \leq \sqrt{2 \mathrm{f} \gamma}
$$

for any $i \neq k \in\{1, \ldots, \mathrm{f}\}$, where $d(\cdot, \cdot)$ is the trace distance $d(\rho, \sigma):=(1 / 2) \cdot \operatorname{Tr}|\rho-\sigma|$ for quantum states $\rho$ and $\sigma$. Thus,
Fannes inequality for mutual information [41, Eq. (5.106)] implies that

$$
\begin{aligned}
& \left|I\left(M_{k} ; \mathcal{A}_{\mathrm{t}} Q_{\mathrm{t}} \mid K=k\right)_{\rho\left(M, Q_{\mathrm{t}}\right)}-I\left(M_{k} ; \mathcal{A}_{\mathrm{t}} Q_{\mathrm{t}} \mid K=i\right)_{\rho\left(M, Q_{\mathrm{t}}\right)}\right| \\
& \leq g(\mathrm{~m}, \gamma),
\end{aligned}
$$

which yields the inequality $(a)$. This finishes the proof of Lemma 4

\section{APPENDIX F}

\section{PROOFS OF CONVERSE BOUNDS (50) AND (51)}

In the following proofs, we use the notations given in Step 1 of Section VI-B and the notation for distributions introduced in the beginning of Appendix E

\section{A. Proof of (50)}

Eq. (50) is proved as follows. Fix $K=k$ and $Q=q$. Applying [41, (4.66)] with $\rho(q)$ and $Y_{k, q}$, we have

$$
\begin{aligned}
& \left(1-P_{\text {err }, k, q}\left(\Psi_{\mathrm{QPIR}}^{(\mathrm{m})}\right)\right)^{1+r} \mathrm{~m}^{r} \\
& \leq \frac{1}{\mathrm{~m}} \sum_{m_{k}=1}^{\mathrm{m}} \operatorname{Tr} \rho\left(m_{k}, q\right)^{1+r} \rho(q)^{-r}
\end{aligned}
$$

for $r \in(0,1)$. Then,

$$
\begin{aligned}
& \frac{1}{\mathrm{~m}} \sum_{m_{k}=1}^{\mathrm{m}} \operatorname{Tr} \rho\left(m_{k}, q\right)^{1+r} \rho(q)^{-r} \leq \frac{1}{\mathrm{~m}} \sum_{m_{k}=1}^{\mathrm{m}} \operatorname{Tr} \rho\left(m_{k}, q\right) \rho(q)^{-r} \\
& =\operatorname{Tr} \rho(q)^{1-r} \leq \max _{\rho} \operatorname{Tr} \rho^{1-r}=\max _{p_{X}} \sum_{x=1}^{\mathrm{d}^{\mathrm{n}}} p_{X=x}^{1-r} \\
& \stackrel{(a)}{=}\left(\prod_{s=1}^{\mathrm{n}} \operatorname{dim} \mathcal{A}_{s}\right)^{r}
\end{aligned}
$$

where the equation $(a)$ is proved from the fact that $\max _{p_{X}} \sum_{x=1}^{\mathrm{d}^{\mathrm{n}}} p_{X=x}^{1-r}$ is achieved by choosing $p_{X}$ as the uniform distribution since the map $y \mapsto y^{1-r}$ is concave. Combining (112) and (113), we have

$$
\left(1-P_{\mathrm{err}, k, q}\left(\Psi_{\mathrm{QPIR}}^{(\mathrm{m})}\right)\right)^{1+r} \leq\left(\frac{\prod_{s=1}^{\mathrm{n}} \operatorname{dim} \mathcal{A}_{s}}{\mathrm{~m}}\right)^{r} .
$$

Let $\left\{\Psi_{\mathrm{QPIR}}^{\left(\mathrm{m}_{\ell}\right)}\right\}_{\ell=1}^{\infty}$ be an arbitrary sequence of QPIR protocols such that the QPIR rate of $\Psi_{\mathrm{QPIR}}^{\left(\mathrm{m}_{\ell}\right)}$ is strictly greater than 1 for any sufficiently large $\ell$, i.e.,

$$
R\left(\Psi_{\mathrm{QPIR}}^{\left(\mathrm{m}_{\ell}\right)}\right)=\frac{\log \mathrm{m}_{\ell}}{\log \mathrm{d}_{\ell}^{\mathrm{n}}}>1,
$$

where $\mathrm{d}_{\ell}$ is the dimension of $\mathcal{A}_{s}(\forall s \in\{1, \ldots, \mathrm{n}\})$ for the protocol $\Psi_{\mathrm{QPIR}}^{\left(\mathrm{m}_{\ell}\right)}$. Then, Eq. (115) implies that $\mathrm{d}_{\ell}^{\mathrm{n}} / \mathrm{m}_{\ell}=$ $\left(\prod_{i=1}^{\mathrm{n}} \operatorname{dim} \mathcal{A}_{i}\right) / \mathrm{m}_{\ell}$ goes to 0 . Hence, from (114), for any $k$ and $q, 1-P_{\mathrm{err}, k, q}\left(\Psi_{\mathrm{QPIR}}^{\left(\mathrm{m}_{\ell}\right)}\right)$ approaches zero. Since

$$
1-P_{\mathrm{err}}\left(\Psi_{\mathrm{QPIR}}^{\left(\mathrm{m}_{\ell}\right)}\right) \leq 1-P_{\mathrm{err}, k, q}\left(\Psi_{\mathrm{QPIR}}^{\left(\mathrm{m}_{\ell}\right)}\right),
$$

we have $1-P_{\mathrm{err}}\left(\Psi_{\mathrm{QPIR}}^{\left(\mathrm{m}_{\ell}\right)}\right) \rightarrow 0$, which implies $(\underline{50})$. 


\section{B. Proof of (51)}

Eq. (51) is proved as follows. Assume that $S_{\text {serv }}\left(\Psi_{\mathrm{QPIR}}^{(\mathrm{m})}\right)=$ 0 and $S_{\text {user }}^{(\mathrm{t})}\left(\Psi_{\mathrm{QPIR}}^{(\mathrm{m})}\right)=0$. We consider the case where arbitrary $K=k, Q=q$, and $\pi \in \mathrm{S}_{\mathrm{n}}$ are fixed. Since Lemma 4 guarantees that the reduced density $\rho\left(m_{k}, q_{\mathrm{t}}\right)$ on $\mathcal{A}_{\mathrm{t}}$ does not depend on $m_{k}$, we have $\rho\left(m_{k}, q_{\mathrm{t}}\right)=\rho\left(q_{\mathrm{t}}\right)$. Applying [41, (4.66)] with $\rho\left(q_{\mathrm{t}}\right) \otimes\left(I / \mathrm{d}^{\mathrm{n}-\mathrm{t}}\right)$ and $Y_{k, q}$, we have

$$
\begin{aligned}
& \left(1-P_{\mathrm{err}, k, q}\left(\Psi_{\mathrm{QPIR}}^{(\mathrm{m})}\right)\right)^{1+r} \mathrm{~m}^{r} \\
& \leq \frac{1}{\mathrm{~m}} \sum_{m_{k}=1}^{\mathrm{m}} \operatorname{Tr} \rho\left(m_{k}, q\right)^{1+r}\left(\rho\left(q_{\mathrm{t}}\right) \otimes \frac{I}{\mathrm{~d}^{\mathrm{n}-\mathrm{t}}}\right)^{-r}
\end{aligned}
$$

for any $r \in(0,1)$. Given $m_{k}$ and $q$, consider the decomposition $\rho\left(m_{k}, q\right)=\sum_{x} p_{x}\left|\psi_{m_{k}, q, x}\right\rangle\left\langle\psi_{m_{k}, q, x}\right|$. Let $\rho\left(q_{\mathrm{t}}, x\right)$ be the reduced density of $\left|\psi_{m_{k}, q, x}\right\rangle\left\langle\psi_{m_{k}, q, x}\right|$ on $\mathcal{A}_{\mathrm{t}}$, i.e., $\rho\left(q_{\mathrm{t}}\right)=\sum_{x} p_{x} \rho\left(q_{\mathrm{t}}, x\right)$. Then,

$$
\begin{aligned}
& \operatorname{Tr} \rho\left(m_{k}, q\right)^{1+r}\left(\rho\left(q_{\mathrm{t}}\right) \otimes \frac{I}{\mathrm{~d}^{\mathrm{n}-\mathrm{t}}}\right)^{-r} \\
& \stackrel{(b)}{\leq} \sum_{x} p_{x} \operatorname{Tr}\left(\left|\psi_{m_{k}, q, x}\right\rangle\left\langle\psi_{m_{k}, q, x}\right|\right)^{1+r}\left(\rho\left(q_{\mathrm{t}}, x\right) \otimes \frac{I}{\mathrm{~d}^{\mathrm{n}-\mathrm{t}}}\right)^{-r} \\
& =\sum_{x} p_{x} \operatorname{Tr}\left|\psi_{m_{k}, q, x}\right\rangle\left\langle\psi_{m_{k}, q, x}\right|\left(\rho\left(q_{\mathrm{t}}, x\right) \otimes \frac{I}{\mathrm{~d}^{\mathrm{n}-\mathrm{t}}}\right)^{-r} \\
& =\mathrm{d}^{r(\mathrm{n}-\mathrm{t})} \sum_{x} p_{x} \operatorname{Tr}\left(\rho\left(q_{\mathrm{t}}, x\right)\right)^{1-r} \stackrel{(c)}{\leq} \mathrm{d}^{2 r(\mathrm{n}-\mathrm{t})},
\end{aligned}
$$

where $(b)$ follows from the application of the inequality

$$
\phi(-r \mid \rho \| \rho):=\log \operatorname{Tr} \rho^{1+r} \rho^{-r} \geq \phi(-r \mid \kappa(\rho) \| \kappa(\rho))
$$

for states $\rho, \rho$, TP-CP map $\kappa$, and $r \in(0,1)$ [41 (5.53)] to the choice $\rho:=\sum_{x} p_{x}|x\rangle\left\langle x|\otimes| \psi_{m_{k}, q, x}\right\rangle\left\langle\psi_{m_{k}, q, x}\right|$, $\rho:=\sum_{x} p_{x}|x\rangle\langle x| \otimes\left(\rho\left(q_{\mathrm{t}}, x\right) \otimes I / \mathrm{d}^{\mathrm{n}-\mathrm{t}}\right)$ on the composite system $\mathcal{X} \otimes \mathcal{A}$, and $\kappa:=\operatorname{Tr}_{\mathcal{X}}$. The last inequality $(c)$ is shown as follows. Since $\rho\left(q_{\mathrm{t}}, x\right)$ is a reduced state of the pure state $\left|\psi_{m_{k}, q, x}\right\rangle$ in $\mathcal{A}_{\mathbf{t}} \otimes \mathcal{A}_{\mathfrak{t} c}$, we have $\operatorname{rank} \rho\left(q_{\mathrm{t}}, x\right) \leq \min \left\{\operatorname{dim} \mathcal{A}_{\mathrm{t}}, \operatorname{dim} \mathcal{A}_{\mathrm{t}^{c}}\right\}=\mathrm{d}^{\mathrm{n}-\mathrm{t}}$, which implies $\operatorname{Tr} \rho\left(q_{\mathrm{t}}, x\right)^{1-r} \leq\left(\mathrm{d}^{\mathrm{n}-\mathrm{t}}\right)^{r}$. Combining (118) and (119), we have

$$
\begin{aligned}
& \left(1-P_{\mathrm{err}, k, q}\left(\Psi_{\mathrm{QPIR}}^{(\mathrm{m})}\right)\right)^{1+r} \\
& =\left(1-P_{\mathrm{err}, k, q}\left(\Psi_{\mathrm{QPIR}}^{(\mathrm{m})}\right)\right)^{1+r} \mathrm{~m}^{r} / \mathrm{m}^{r} \\
& \leq \frac{\mathrm{d}^{2 r(\mathrm{n}-\mathrm{t})}}{\mathrm{m}^{r}}=\left(\frac{\mathrm{d}^{2(\mathrm{n}-\mathrm{t})}}{\mathrm{m}}\right)^{r}
\end{aligned}
$$

Let $\left\{\Psi_{\mathrm{QPIR}}^{\left(\mathrm{m}_{\ell}\right)}\right\}_{\ell=1}^{\infty}$ be an arbitrary sequence of QPIR protocols such that the QPIR rate greater than $2(n-t) / n$ for any sufficiently large $\ell$, i.e.,

$$
R\left(\Psi_{\mathrm{QPIR}}^{\left(\mathrm{m}_{\ell}\right)}\right)=\frac{\log \mathrm{m}_{\ell}}{\log \mathrm{d}_{\ell}^{\mathrm{n}}}>\frac{2(\mathrm{n}-\mathrm{t})}{\mathrm{n}},
$$

which is equivalent to

$$
\frac{\log m_{\ell}}{\log d_{\ell}^{2(n-t)}}>1
$$

Here, $\mathrm{d}_{\ell}$ is the dimension of $\mathcal{A}_{s}(\forall s \in\{1, \ldots, \mathrm{n}\})$ for the protocol $\Psi_{\mathrm{QPIR}}^{\left(\mathrm{m}_{\ell}\right)}$. From (122), $\mathrm{d}_{\ell}^{2(\mathrm{n}-\mathrm{t})} / \mathrm{m}_{\ell}$ goes to 0 , and then from (120), the probability $1-P_{\mathrm{err}, k, q}\left(\Psi_{\mathrm{QPIR}}^{\left(\mathrm{m}_{\ell}\right)}\right)$ approaches 0 . Since

$$
1-P_{\mathrm{err}}\left(\Psi_{\mathrm{QPIR}}^{\left(\mathrm{m}_{\ell}\right)}\right) \leq 1-P_{\mathrm{err}, k, q}\left(\Psi_{\mathrm{QPIR}}^{\left(\mathrm{m}_{\ell}\right)}\right),
$$

we have $1-P_{\mathrm{err}}\left(\Psi_{\mathrm{QPIR}}^{\left(\mathrm{m}_{\ell}\right)}\right) \rightarrow 0$, which implies $\underline{51}$.

\section{REFERENCES}

[1] B. Chor, O. Goldreich, E. Kushilevitz, and M. Sudan, "Private information retrieval," Journal of the ACM, 45(6):965-981, 1998.

[2] A. Beimel and Y. Stahl, "Robust information-theoretic private information retrieval," Proceedings of the 3rd International Conference on Security in Communication Networks (SCN'02), pp. 326-341, 2003.

[3] S. Yekhanin, "Towards 3-query locally decodable codes of subexponential length," 39th STOC, 2007, pp. 266-274.

[4] C. Devet, I. Goldberg, and N. Heninger, "Optimally Robust Private Information Retrieval,” 21st USENIX Security Symposium, August 2012.

[5] H. Sun and S. Jafar, "The capacity of private information retrieval," IEEE Transactions on Information Theory, vol. 63, no. 7, pp. 40754088, 2017.

[6] T. H. Chan, S.-W. Ho, and H. Yamamoto, "Private information retrieval for coded storage," in Proceedings of 2015 IEEE International Symposium on Information Theory (ISIT), pp. 2842-2846, June 2015.

[7] H. Sun and S. Jafar, "The Capacity of Symmetric Private Information Retrieval,” 2016 IEEE Globecom Workshops (GC Wkshps), Washington, DC, 2016, pp. 1-5.

[8] H. Sun and S. Jafar, "The capacity of robust private information retrieval with colluding databases," IEEE Transactions on Information Theory, vol. 64, no. 4, pp. 2361-2370, 2018.

[9] K. Banawan and S. Ulukus, "The Capacity of Private Information Retrieval from Coded Databases," IEEE Transactions on Information Theory, vol. 64, no. 3, 2018.

[10] R. Freij-Hollanti, O. W. Gnilke, C. Hollanti, and D. A. Karpuk, "Private information retrieval from coded databases with colluding servers," SIAM J. Appl. Algebra Geometry, vol. 1, no. 1, pp. 647-664, 2017.

[11] S. Kumar, H.-Y. Lin, E. Rosnes, and A. Graell i Amat, "Achieving maximum distance separable private information retrieval capacity with linear codes," IEEE Transactions on Information Theory, vol. 65, no. 7, pp. 4243-4273, 2019.

[12] H.-Y. Lin, S. Kumar, E. Rosnes, and A. Graell i Amat, "An MDSPIR capacity-achieving protocol for distributed storage using non-MDS linear codes," Proc. IEEE Int. Symp. Inf. Theory, June 17-22, 2018.

[13] C. Tian, H. Sun and J. Chen, "Capacity-Achieving Private Information Retrieval Codes With Optimal Message Size and Upload Cost," IEEE Transactions on Information Theory, vol. 65, pp. 7613-7627, 2019.

[14] C. Tian, H. Sun and J. Chen, "A Shannon-Theoretic Approach to the Storage-Retrieval Tradeoff in PIR Systems," Proceedings of 2018 IEEE International Symposium on Information Theory (ISIT), pp. 1904-1908, June 2018.

[15] Q. Wang and M. Skoglund, "Symmetric private information retrieval for MDS coded distributed storage," Proceedings of 2017 IEEE International Conference on Communications (ICC), pp. 1-6, May 2017.

[16] Q. Wang and M. Skoglund, "Secure Symmetric Private Information Retrieval from Colluding Databases with Adversaries," 2017 55th Annual Allerton Conference on Communication, Control, and Computing (Allerton), pp. 1083-1090, 2017.

[17] R. Tandon, "The capacity of cache aided private information retrieval," Proceedings of 2017 55th Annual Allerton Conference on Communication, Control, and Computing (Allerton), pp. 1078-1082, 2017.

[18] K. Banawan and S. Ulukus, "The capacity of private information retrieval from byzantine and colluding databases," IEEE Transactions on Information Theory, vol. 65, no. 2, pp. 1206-1219, 2019.

[19] L. Holzbaur, R. Freij-Hollanti, J. Li, C. Hollanti, "Towards the Capacity of Private Information Retrieval from Coded and Colluding Servers," arXiv:1903.12552 [cs.IT], 2019.

[20] I. Kerenidis and R. de Wolf. "Exponential lower bound for 2-query locally decodable codes via a quantum argument," Proceedings of 35th ACM STOC, pp. 106-115, 2003.

[21] I. Kerenidis and R. de Wolf, "Quantum symmetrically-private information retrieval," Information Processing Letters, vol. 90, pp. 109-114, 2004.

[22] L. Olejnik, "Secure quantum private information retrieval using phaseencoded queries," Physical Review A 84, 022313, 2011. 
[23] F. Le Gall, "Quantum Private Information Retrieval with Sublinear Communication Complexity," Theory of Computing, 8(16):369-374, 2012.

[24] Ä. Baumeler and A. Broadbent, "Quantum Private Information Retrieval has linear communication complexity," Journal of Cryptology, vol. 28, issue 1, pp. 161-175, 2015.

[25] I. Kerenidis, M. Laurière, F. Le Gall, and M. Rennela, "Information cost of quantum communication protocols," Quantum information \& computation, 16(3-4):181-196, 2016.

[26] D. Aharonov, Z. Brakerski, K.-M. Chung, A. Green, C.-Y. Lai, O. Sattath, "On Quantum Advantage in Information Theoretic Single-Server PIR,” In: Ishai Y., Rijmen V. (eds) EUROCRYPT 2019, Springer, Cham, vol. 11478, 2019.

[27] S. Song and M. Hayashi, "Capacity of Quantum Private Information Retrieval with Multiple Servers," IEEE Transactions on Information Theory, DOI:10.1109/TIT.2020.3022515, in press.

[28] S. Song and M. Hayashi, "Capacity of Quantum Private Information Retrieval with Collusion of All But One of Servers," IEEE Journal on Selected Areas in Information Theory, vol. 2, no. 1, pp. 380-390, 2021.

[29] M. Allaix, L. Holzbaur, T. Pllaha, and C. Hollanti, "Quantum Private Information Retrieval From Coded and Colluding Servers," IEEE Journal on Selected Areas in Information Theory, vol. 1, no. 2, pp. 599-610, 2020.

[30] M. Rabin, "How to exchange secrets by oblivious transfer", Tech. Memo TR-81, Aiken Computation Laboratory, Harvard University, 1981

[31] Y. Gertner, Y. Ishai, E. Kushilevitz, and T. Malkin. "Protecting data privacy in private information retrieval schemes," Journal of Computer and Systems Sciences, 60(3):592-629, 2000. Earlier version in STOC 98.

[32] C. Bennett and S. Wiesner, "Communication via one- and two-particle operators on Einstein-Podolsky-Rosen states," Physical Review Letters, 69 (20): 2881, 1992.

[33] C. H. Bennett, G. Brassard, C. Crépeau, R. Jozsa, A. Peres, and W. K. Wootters, "Teleporting an unknown quantum state via dual classical and Einstein-Podolsky-Rosen channels," Physical Review Letters, 70(13):1895-1899, 1993.

[34] A. R. Calderbank, E. M. Rains, P. W. Shor, and N. J. A. Sloane, "Quantum error correction via codes over gf (4)," IEEE Transactions on Information Theory, vol. 44, no. 4, pp. 1369-1387, 1998.

[35] A. Ashikhmin and E. Knill, "Nonbinary quantum stabilizer codes," IEEE Transactions on Information Theory, vol. 47, no. 7, pp. 3065-3072, 2001.

[36] A. Ketkar, A. Klappenecker, S. Kumar and P. Sarvepalli, "Nonbinary stablizer codes over finite fields," IEEE Transactions on Information Theory, vol. 52, no. 11, pp. 4892-4914, 2006.

[37] M. Hayashi, Group Representation for Quantum Theory, Cham, Switzerland: Springer, 2017.

[38] F. J. MacWilliams and N. J. A. Sloane, The Theory of Error-Correcting Codes, Amsterdam: North-Holland, 1977.

[39] R. Lidl and H. Niederreiter, Finite Fields (2nd ed., Encyclopedia of Mathematics and its Applications), Cambridge: Cambridge University Press, 1996

[40] M. A. Nielsen and I. L. Chuang, Quantum computation and quantum information, Cambridge: Cambridge University Press, 2000.

[41] M. Hayashi, Quantum Information Theory: Mathematical Foundation, Graduate Texts in Physics, Springer, (Second edition of Quantum Information: An Introduction, Springer), 2017.

[42] L. H. Ozarow and A. D. Wyner, "Wire-tap channel II," AT \& T Bell Labs. Tech. J., vol. 63, pp. $2135-2157,1984$.

[43] N. Cai and M. Hayashi, "Secure Network Code for Adaptive and Active Attacks with No-Randomness in Intermediate Nodes," IEEE Transactions on Information Theory, vol. 66, 1428-1448, 2020.

[44] N. Cai and R. W. Yeung, "Secure Network Coding on a Wiretap Network," IEEE Trans. Inform. Theory, vol. 57, no. 1, 424-435, 2011.

[45] R. C. Singleton, "Maximum distance q-nary codes," IEEE Transactions on Information Theory, 10 (2): 116-118.

[46] C. H. Bennett, P. W. Shor, J. A. Smolin, and A. V. Thapliyal, "Entanglement-assisted classical capacity of noisy quantum channels," Physical Review Letters, 83(15):3081-3084, 1999.

[47] S. Song and M. Hayashi, "Capacity of Quantum Private Information Retrieval with Colluding Servers," Proceedings of 2020 IEEE International Symposium on Information Theory (ISIT), pp. 1077-1082, 2020
Seunghoan Song (GS'20-M'21) received the B.E. degree from Osaka University, Japan, in 2017 and the M.Math. and Ph.D. degrees in mathematical science from Nagoya University, Japan, in 2019 and 2020, respectively. He is a research fellow of the Japan Society of the Promotion of Science (JSPS) from 2020. He is currently a JSPS postdoctoral fellow at the Graduate School of Mathematics, Nagoya University. He awarded the School of Engineering Science Outstanding Student Award at Osaka University in 2017 and Graduate School of Mathematics Award for Outstanding Masters Thesis at Nagoya University in 2019. His research interests include classical and quantum information theory and its applications to secure communication protocols.

Masahito Hayashi (M'06-SM'13-F'17) was born in Japan in 1971. He received the B.S. degree from the Faculty of Sciences in Kyoto University, Japan, in 1994 and the M.S. and Ph.D. degrees in Mathematics from Kyoto University, Japan, in 1996 and 1999, respectively. He worked in Kyoto University as a Research Fellow of the Japan Society of the Promotion of Science (JSPS) from 1998 to 2000, and worked in the Laboratory for Mathematical Neuroscience, Brain Science Institute, RIKEN from 2000 to 2003, and worked in ERATO Quantum Computation and Information Project, Japan Science and Technology Agency (JST) as the Research Head from 2000 to 2006. He also worked in the Superrobust Computation Project Information Science and Technology Strategic Core (21st Century COE by MEXT) Graduate School of Information Science and Technology, The University of Tokyo as Adjunct Associate Professor from 2004 to 2007. He worked in the Graduate School of Information Sciences, Tohoku University as Associate Professor from 2007 to 2012. In 2012, he joined the Graduate School of Mathematics, Nagoya University as Professor. Also, he was appointed in Centre for Quantum Technologies, National University of Singapore as Visiting Research Associate Professor from 2009 to 2012 and as Visiting Research Professor from 2012 to now. He worked in Center for Advanced Intelligence Project, RIKEN as a Visiting Scientist from 2017 to 2020. He worked in Shenzhen Institute for Quantum Science and Engineering, Southern University of Science and Technology, Shenzhen, China as a Visiting Professor from 2018 to 2020, and in Center for Quantum Computing, Peng Cheng Laboratory, Shenzhen, China as a Visiting Professor from 2019 to 2020. In 2020, he joined Shenzhen Institute for Quantum Science and Engineering, Southern University of Science and Technology, Shenzhen, China as Chief Research Scientist. In 2011, he received Information Theory Society Paper Award (2011) for "Information-Spectrum Approach to Second-Order Coding Rate in Channel Coding". In 2016, he received the Japan Academy Medal from the Japan Academy and the JSPS Prize from Japan Society for the Promotion of Science.

In 2006, he published the book "Quantum Information: An Introduction" from Springer, whose revised version was published as "Quantum Information Theory: Mathematical Foundation" from Graduate Texts in Physics, Springer in 2016. In 2016, he published other two books "Group Representation for Quantum Theory" and "A Group Theoretic Approach to Quantum Information" from Springer. He is on the Editorial Board of International Journal of Quantum Information and International Journal On Advances in Security. His research interests include classical and quantum information theory and classical and quantum statistical inference. 